\title{
Emotion Regulation and Psychological and Physical Health during a Nationwide COVID-19 Lockdown
}

\author{
Rachel S. T. Low ${ }^{1}$, Nickola C. Overall ${ }^{2}$, Valerie T. Chang ${ }^{2}$, \\ Annette M. E. Henderson² \& Chris G. Sibley ${ }^{2}$ \\ ${ }^{1}$ School of Social Sciences and Public Policy, Auckland University of Technology \\ ${ }^{2}$ School of Psychology, University of Auckland
}

Low, R.S.T., Overall, N.C., Chang, V.T., Henderson A.M.E., Sibley, C.G. (in press).

Emotion Regulation and Psychological and Physical Health during a Nationwide COVID-19 Lockdown. Emotion.

Author Note: Correspondence regarding this manuscript can be directed to Rachel S. T. Low, School of Social Sciences \& Public Policy, Auckland University of Technology, Private Bag 92006, Auckland 1142, New Zealand, Email: rachel.low@aut.ac.nz.

Funding Statement: Study 1 was supported by a Royal Society of New Zealand Marsden Fund grant (UOA1712) awarded to Nickola C. Overall and Annette M. E. Henderson. Study 2 used data from The New Zealand Attitudes and Values Study, which is supported by a grant from the Templeton Religion Trust (TRT0196). The funders had no role in the preparation of the manuscript or the decision to publish. 


\begin{abstract}
The current research tests the links between emotion regulation and psychological and physical health during the COVID-19 pandemic. In Study 1, parents $(N=365)$ who had reported on their psychological and physical health prior to the pandemic completed the same health assessments along with their use of emotion regulation strategies when confined in the home with their school-aged children during a nationwide lockdown. In Study 2, individuals $(N=1,607)$ from a nationally representative panel study completed similar measures of psychological and physical health and use of emotion regulation strategies 1-year prior to the lockdown and then again during the lockdown. Accounting for pre-pandemic psychological health, greater rumination and emotional suppression were independently associated with poorer psychological health (greater depressive symptoms and psychological distress, lower emotional and personal well-being), even when controlling for the emotional challenges of the pandemic (emotion control difficulties, perceived support; Studies 1 and 2) and a range of demographic covariates (Study 2). Greater rumination was also associated with greater fatigue in both studies, but greater rumination and emotional suppression were only independently associated with poorer perceptions of physical health in Study 2. The results for cognitive reappraisal were mixed; positive associations with personal well-being and general health only emerged in Study 2 . The results provide evidence that key models in affective science help explain differences in psychological and physical health within the throes of a real-world demanding context, and thus offer targets to help facilitate health and resilience during the pandemic (and other crises).
\end{abstract}

Keywords: COVID-19, emotion regulation, stress, health, well-being 


\section{Emotion Regulation and Psychological and Physical Health during a Nationwide COVID-19 Lockdown}

The COVID-19 pandemic poses considerable challenges that may undermine health and well-being. Mandatory lockdowns to contain the spread of the virus require people to isolate in their homes and manage work, childcare, economic loss and the stress of the pandemic with little support and physical contact with others outside their household. As the pandemic emerged, experts forecasted a rise in mental health issues (Holmes et al., 2020) and many people reported heightened levels of stress, anxiety, and depression (e.g., Joiner \& Twenge, 2020) and demonstrated declines in well-being (Zacher \& Rudolph, 2020) in the initial months of the pandemic (also see Aknin et al., in press). Yet, based on typical trajectories of health following loss and disasters, resilience experts have argued that most people will likely sustain health and well-being (Chen \& Bonanno, 2020), which fit with some studies finding only small average drops in health and well-being during a nationwide COVID-19 lockdown (Sibley et al., 2020) and relative resilience in well-being and life satisfaction after the early months of the pandemic (Aknin et al., in press).

Examinations of average levels of well-being fail to identify who may experience poorer psychological and physical health versus who may remain relatively resilient. Given the cumulative challenges of the pandemic, identifying the factors that may risk versus protect psychological and physical health is of utmost importance (Zacher \& Rudolph, 2020). One central factor that should influence the degree to which the challenges of the pandemic negatively affect health and well-being is emotion regulation, which involves the conscious or unconscious efforts to influence the experience, expression, duration, and magnitude of emotions (Gross, 1998). In particular, theories of emotion regulation highlight that, rather than simply encountering emotional challenges, the ways people regulate emotions during challenging situations should influence psychological and physical health (Gross, 2015; Gross \& Muñoz, 1995). The current research tests the links between the enactment of 
different emotion regulation strategies during the COVID-19 pandemic and relative levels of psychological and physical health.

We focused on three key emotion regulation strategies that have been reliably associated with health outcomes, and that theoretical and empirical work indicates disrupts psychological and social processes that should be important in sustaining health and wellbeing during the pandemic. Rumination, involving fixating on the sources and experience of distress, has been shown to be critical to the development and maintenance of depression (Nolen-Hoeksema, 1991). Emotional suppression, involving inhibiting the expression and/or experience of emotions, tends to prolong negative emotions, interfere with problem solving, and impede social connections, which should increase the risk of poorer psychological and physical health (Gross \& Muñoz, 1995). By contrast, cognitive reappraisal, involving changing the way one thinks about the situation (Gross, 1998), is theorized to reduce distress, foster optimism for the future, and help buffer emotionally challenging situations (see Marroquín et al., 2017; Quoidbach et al., 2010).

To test whether the use of rumination, emotional suppression and cognitive reappraisal during the pandemic was associated with relative levels of psychological and physical health, we drew upon two samples of participants who completed assessments of psychological and physical health prior to the COVID-19 pandemic. Participants in both Study $1(N=365)$ and Study $2(N=1,607)$ completed the same health assessments along with assessments of emotion regulation during a mandatory nationwide COVID-19 lockdown. These repeated assessments allowed us to test whether emotion regulation during this acute challenging context was associated with relative levels of psychological and physical health accounting for health prior to the pandemic.

\section{Emotion Regulation and Psychological and Physical Health}

In this section, we summarize a body of work providing evidence that rumination, emotional suppression, and cognitive reappraisal are associated with psychological and 
physical health. We also review evidence of how these emotion regulation strategies disrupt personal and interpersonal processes that are likely to be important in sustaining psychological and physical health in the emotionally challenging context of the pandemic.

Rumination. Greater trait rumination is associated with greater negative affect (Aldao et al., 2010), greater depressive symptoms and poorer mental health (Fritz, 1999), and is generally associated with poorer physical health, such as increased and prolonged cortisol reactivity (Zoccola et al., 2008), poorer cardiovascular recovery from stress (Radstaak et al., 2011), and lower self-reported health (Thomsen et al., 2004).

Theories of rumination point to various mechanisms for these poor health outcomes. Rumination involves repetitive thinking about sadness and the circumstances related to one's sadness, which is likely to intensify and prolong negative emotions, and maintain depressed mood (Conway, 2000; Nolen-Hoeksema, 1991). People who report greater rumination also are more self-focused and perceive the self and others more negatively within social interactions (Mellings \& Alden, 2000; Takano et al., 2011). Greater fixation on negative thoughts and emotions also depletes cognitive resources and so likely reduces the degree to which people can problem solve (McNally, 1995). Indeed, rumination is associated with difficulties with problem solving (Clore \& Gasper, 2000; Watkins \& Brown, 2002), reduced motivation (Lyubomirsky et al., 1999), less instrumental behaviors (Nolen-Hoeksema, 1998), and increased reckless and unhealthy behaviors (e.g., alcohol abuse; Nolen-Hoeksema, 2004).

Taken together, this prior theoretical and empirical work indicate that greater rumination during the COVID-19 pandemic and lockdowns may exacerbate negative affect, negatively tinge views of social interactions, interfere with problem solving and motivation, and interfere with people's ability to engage in activities that promote health (e.g., exercise, meditation). Thus, greater use of rumination when experiencing the challenges of the COVID-19 pandemic, such as a mandatory nationwide lockdown, is likely to be associated with poorer psychological and physical health (accounting for pre-pandemic health). 
Emotional suppression. Laboratory experiments, daily sampling paradigms, behavioral observation and longitudinal designs provide consistent evidence that emotional suppression poses a risk to psychological and physical health. Greater use of emotional suppression is concurrently associated with higher negative affect and lower positive affect (Brans et al., 2013; Impett et al., 2012; Low et al., 2017) and predicts longitudinal increases in anxiety and depressive symptoms (Cameron \& Overall, 2018; Gross \& John, 2003; Kashdan et al., 2008; Wenzlaff \& Luxton, 2003). Greater use of emotional suppression increases physiological threat responses (Gross \& Levenson, 1993, 1997; Peters et al., 2014; Peters \& Jamieson, 2016), and is linked to higher levels of inflammation (Appleton et al., 2013), greater risk of cardiovascular disease (Graves et al., 1994) and faster progression of disease (Tamagawa et al., 2013). Similar avoidance-oriented coping strategies, such as attempts to avoid stressor-related thoughts, also are associated with more negative outcomes, such as increased PTSD symptoms and psychological distress (Glass et al., 2009; Kamo et al., 2011).

A variety of mechanisms for why emotional suppression risks poorer health and wellbeing have been identified. Attempts to inhibit or suppress negative emotions tend to exacerbate negative emotions and increase the accessibility of negative thoughts (Dalgleish et al., 2009; Kelly \& Kahn, 1994). Emotional suppression also requires substantial effort, reducing cognitive and regulatory resources that could otherwise be directed towards problem solving (Goldberg \& Grandey, 2007; Richards \& Gross, 1999, 2000). These reductions in resources are likely one reason why emotional suppression interferes with people's ability to overcome challenges in their daily lives, such as resolving relationship conflicts or sustaining motivation to persevere with personal goals (Low et al., 2017, 2019; Thomson et al., 2018). Finally, suppressing emotions tends to reduce social connectedness (English \& John, 2013; Srivastava et al., 2009), which is critical for sustaining health and well-being. 
In sum, a range of prior theoretical and empirical work indicate that people who enact emotional suppression within emotionally relevant contexts are susceptible to prolonged negativity, poorer problem solving, and reduced social connectedness. Given the importance of managing negative emotions, sustaining social connections, and problem solving in the challenging context of the pandemic, the use of emotional suppression during COVID-19 lockdowns is likely to be associated with relatively poorer psychological and physical health (accounting for pre-pandemic psychological and physical health).

Cognitive reappraisal. The detrimental effects typically associated with rumination and emotional suppression are why these are often referred to as "maladaptive" emotion regulation strategies. Cognitive reappraisal, in contrast, is generally thought to be an "adaptive" strategy, which is supported by research showing cognitive reappraisal is associated with more positive health and well-being outcomes. Greater use of cognitive reappraisal is associated with lower negative affect, greater well-being, and lower depressive symptoms (Cludius et al., 2020; Gross \& John, 2003; Hopp et al., 2011; Mauss et al., 2005). Moreover, unlike rumination and emotional suppression, prior research indicates that cognitive reappraisal does not increase sympathetic activation of the cardiovascular system (Mauss et al., 2007), is associated with lower levels of inflammation (Appleton et al., 2013) and greater levels of heart rate variability, which is an indicator of adaptive emotion regulation (Denson et al., 2011). Similar types of approach-oriented coping strategies, such as positive reframing or seeking social support, are also associated with greater subjective wellbeing and post-traumatic growth (Kishore et al., 2008; Mesidor \& Sly, 2016).

There are several reasons why cognitive reappraisal is likely to be more beneficial for health and well-being. According to Gross' (1998) process model of emotion regulation, cognitive reappraisal is typically enacted at the start of the emotion generation process and should therefore be more effective at reducing the subjective experience and physiological signs of negative emotions. Experimentally induced cognitive reappraisal produces less 
emotional reactivity to stressors (Richards \& Gross, 2000), and does not undermine cognitive and regulatory resources as emotional suppression does (Butler et al., 2003; Richards \& Gross, 2000). By reducing the intensity and duration of negative emotions, and leaving cognitive and regulatory resources intact, people should be more effective at dealing with and solving emergent challenges (e.g., Low et al., 2019) and sustaining psychological health (Smith et al., 2021). Thus, compared to rumination and emotional suppression, people who use cognitive reappraisal during the pandemic should be less likely to experience poorer psychological and physical health (accounting for pre-pandemic health).

\section{Current Research and Contributions}

An array of expert commentary and rapid research have warned the challenges of the COVID-19 pandemic may risk health and well-being, but the potential health outcomes of the current crisis will not be uniform. In the current research, we apply affective science to examine a key factor - emotion regulation - that should play an important role in relative levels of psychological and physical health while faced with the challenges of COVID-19 lockdowns. Rumination and emotional suppression are theorized (with empirical support) to disrupt a range of personal and interpersonal processes in emotionally challenging situations that should be critical to successfully traversing the pandemic and lockdowns, including exacerbating distress, interfering with problem solving, creating more negative evaluations or distance within social interactions, and undermining motivation. Cognitive reappraisal, by contrast, may have the opposite and more beneficial effects by facilitating equanimity and promoting problem solving. Thus, testing the links between emotion regulation and psychological and physical health in the midst of an unprecedented challenge puts to the test whether key models in affective science provide insight into health risk versus resilience when emotion regulation processes should really matter.

We conducted this 'real-world' test in two studies that examined emotion regulation during an acute context that was likely challenging for most people-a 5-week mandatory 
nationwide lockdown in which people were confined to their home. This Level 4 lockdown in New Zealand (NZ) was one of the strictest legally enforced lockdowns in the world. ${ }^{1}$ During the 5-week lockdown (March 26 to April 27, 2020), people were legally required to stay within their immediate household 'bubbles' at all times with no physical contact with anyone outside their home. The only exceptions allowed one person to leave the house to gather essential resources (e.g., groceries, medicine) or exercise outside within local neighborhoods as long as strict physical distancing from others outside the immediate household was maintained. In both studies, we assessed people's enactment of emotion regulation strategies during the lockdown to capture the degree to which participants enacted these strategies in the midst of a novel, emotionally relevant, and challenging situation.

In addition to offering the first examination of rumination, emotional suppression and cognitive appraisal in the throes of the COVID-19 pandemic (to date), by leveraging ongoing longitudinal studies, both studies also extended the majority of examinations of the effects of the pandemic on psychological and physical health. Despite an eruption of pandemic-related research, most studies (to date) present cross-sectional examinations that do not account for psychological and physical health preceding the pandemic. By drawing on samples that had completed health assessments prior to the pandemic, and then assessing emotion regulation and health during the lockdown, we tested whether emotion regulation in the midst of a current, acute challenge was associated with relative residual changes in psychological and physical health. By accounting for psychological and physical health prior to the pandemic, the design in both Study $1(N=365)$ and Study $2(N=1607)$ provides a strong test of whether emotion regulation enacted during the COVID-19 lockdown was associated with the relative risk versus resilience of psychological and physical health during the pandemic.

If models of emotion regulation are relevant to explaining health and well-being within acute challenging contexts, then participants who enact greater rumination and emotional suppression to regulate negative emotions during the lockdown should be more 
likely to experience poorer psychological and physical health. By contrast, participants who enact greater cognitive reappraisal should be less likely to experience poorer psychological and physical health. To provide further evidence for the unique effect of each emotion regulation strategy and ensure that the results were not simply the result of the emotional challenges of the pandemic, we controlled for the shared associations across each emotion regulation strategy (and thus the general need to enact emotion regulation). Furthermore, in both studies we ran a range of additional analyses controlling for difficulties in controlling emotions (and thus the general challenge of regulating emotions), stress across life domains (and thus the overall difficulty of the current context), and perceived support (and thus the degree to which the challenge of the pandemic was eased by support from others).

\section{Study 1}

In Study 1, adults who were part of an ongoing longitudinal study were invited to take part in an online survey assessing their experiences during the nationwide lockdown. Prior to the pandemic, participants had completed assessments of their psychological health (depressive symptoms, emotional well-being) and physical health (energy [low fatigue], general health). During the mandatory lockdown, participants completed repeat assessments of their psychological and physical health and reported on their use of rumination, emotional suppression, and cognitive reappraisal during the lockdown. By leveraging a pre-pandemic study, this design allowed us to examine whether the use of different emotion regulation strategies during the lockdown were associated with residual changes in psychological and physical health (i.e., controlling for pre-pandemic psychological and physical health).

\section{Method}

\section{Participants}

Sample size was determined by the pool of participants $(N=468)$ who had already participated in an ongoing study. Participation in the study prior to the COVID-19 pandemic involved families (two parents and one child) completing online questionnaires and attending 
a laboratory session. Parents who continued to live together and reside in NZ were emailed an invitation to complete a questionnaire during the 5-week nationwide Level 4 lockdown. The questionnaire was sent two weeks into the lockdown and open for completion until the end of the lockdown (between April 8 and 27, 2020). Participants received \$NZ25 for completing the lockdown questionnaire. Of the 468 parents who were contacted, $78 \%$ agreed to participate providing a sample of 365 individuals ( 201 females, 163 males, 1 gender diverse), of which 314 were drawn from the same family $(N=157$ dyads, 51 individuals whose partners did not complete the lockdown questionnaire). Participants who completed versus did not complete the lockdown assessment did not differ in pre-lockdown measures of psychological or physical health ( $t \mathrm{~s}<1.57, p \mathrm{~s}>.12$; see Online Supplemental Material $[\mathrm{OSM}]$ ). Power sensitivity analyses accounting for the dependence in the data (Ackerman et al., 2016) indicate high power (.98) to detect small $(r=.20)$ associations between emotion regulation during the lockdown and psychological and physical health.

Parents were on average 37.06 years old $(S D=4.85)$, involved in long-term marital relationships $(M=11.85$ years, $S D=4.54 ; 86 \%$ married, $14 \%$ cohabiting but not married $)$ with an average of two children $(M=2.26, S D=0.87)$. Participants identified as Māori (5.8\%), Pacific Nations (5.5\%), Indian (4.9\%), Asian (10.7\%), non-NZ European (12.6\%), NZ European/Pākehā (55.9\%) and Other (4.1\%). Participants covered a range of income brackets as they entered the pandemic prior to lockdown [per annum NZD; 35.1\% $<40,000$, $11.5 \% 41-60,000,17.5 \% 61-80,000,16.2 \% 81-100,000,19.7 \%>100,000]$. Around a third lost work $(N=112 ; 31 \%)$ and/or income $(N=122 ; 34 \%)$ due to COVID-19, which is evident in change in income groups during the lockdown $[19.4 \%<40,000,15.7 \% 41-60,000,24.1 \%$ $61-80,000,24.1 \% 81-100,000,25.9 \%>100,000]$. See OSM for further sample information.

\section{Procedure and Materials}

The study was approved by the [removed for review] ethics committee. Informed consent was obtained. Prior to the lockdown, all families had completed an initial assessment 
phase, and 78 of the families who completed the lockdown questionnaire had completed a second assessment phase. At both prior assessment phases, parents completed an online questionnaire assessing their psychological and physical health, which were used as baseline measures to examine residual change in psychological and physical health during lockdown. For the current project, we drew on the most recent assessment of health completed, which was on average 207 days $(S D=128$; range 25-658) before the lockdown assessment. Accounting for days between the pre-pandemic and lockdown assessments did not alter the results (see OSM). The same measures of psychological and physical health were included in the lockdown questionnaire along with additional measures assessing emotion regulation strategies, emotion control difficulties, stress across life domains, and perceived support during the lockdown. Completion of the questionnaire occurred on average 20 days $(S D=4$; range $=14-33$ ) into the lockdown. Measures and primary data to replicate the analyses are available here: $\underline{\text { https://osf.io/e5ftj/?view_only=eeccf5b10c1d486782ef0c278443e4d0 }}$

\section{Psychological and Physical Health Prior to and During Lockdown}

Depressive symptoms. Prior to the pandemic, participants completed the 20 -item Centre of Epidemiological Studies Depression Scale (CES-D; Radloff, 1977), which assesses the frequency of depressive symptoms experienced during the past week (e.g., "I felt depressed"; "I felt that everything I did was an effort"; 0 = Rarely or none of the time (less than one day) to $3=$ Most or all of the time (5-7 days)). To reduce burden of participation during the lockdown, we used a shorter 10-item version of the CES-D, which has shown satisfactory test-retest correlations and good predictive accuracy (Andresen et al., 1994). Participants rated each item according to how they felt and behaved during the lockdown (e.g., "I felt depressed"; $0=$ Rarely or none of the time (less than one day per week) to $3=$ Most or all of the time (5-7 days per week)). We generated equivalent measures of depressive symptoms prior to and during the lockdown by summing the items included in the 10-item 
CES-D prior to $(\alpha=.86)$ and during $(\alpha=.83)$ lockdown. Higher scores out of 30 indicate the presence of more depressive symptoms.

At each assessment phase, participants also completed the RAND-36 Item Health Survey 1.0 (Ware et al., 1980), which assesses both psychological and physical health. We focused on the global measures of health from the RAND-36 survey that along with depressive symptoms provided two broad indicators of psychological (depressive symptoms, emotional well-being) and physical health (energy, general health; see OSM for analyses on more specific indices that illustrated similar patterns as reported below). In the pre-lockdown assessments, participants rated each item according to their experiences over the past four weeks. In the lockdown assessments, participants rated each item according to their experiences during the lockdown. Using the scoring outlined by RAND (n.d.), all the items were recoded to $0-100$ scales and then averaged so that higher scores (out of 100) reflect better psychological and physical health.

Emotional well-being. Participants rated five items according to how often $(1=$ None of the time, $5=$ All of the time) they experienced indices of emotional well-being (e.g., "felt calm and peaceful" (reversed-coded; Pre-Lockdown $\alpha=.85$, Lockdown $\alpha=.84$ ).

Energy (Low Fatigue). Participants rated four items according to how often ( 1 = None of the time, 5 = All of the time) they experienced energy/fatigue (e.g., "felt full of life", "felt worn out" (reversed-coded; Pre-Lockdown $\alpha=.83$, Lockdown $\alpha=.81$ ).

General Health. Physical health was assessed using five items such as "In general, would you say your health is... $(1=$ Poor, $5=$ Excellent $)$, and "I seem to get sick a little easier than most people (reversed-coded; 1 = Definitely false, 5 = Definitely true; PreLockdown $\alpha=.79$, Lockdown $\alpha=.78$ ).

\section{Emotion Regulation During Lockdown}

Participants were asked to think about how they thought, felt, and behaved during the lockdown, and rated a series of items used in prior research to assess rumination, emotional 
suppression, and cognitive appraisal during specific emotionally relevant contexts. Items were averaged to index each emotion regulation strategy, with higher scores indicating greater enactment of rumination, emotional suppression, or cognitive reappraisal.

Rumination. Participants rated four items: "I kept thinking about how sad or angry I was feeling", "I kept thinking "Why am I feeling or reacting this way?"”, "I kept thinking about all of the problems we had", "I kept thinking about the same things over and over" $(1=$ Not at all, $7=$ Very much; $\alpha=.86$ ). These items were derived from prior theory and research on the components of ruminative response style associated with depressed mood, including an excessive tendency to focus on potential causes, meaning, and consequences of depressed mood (Gratz \& Roemer, 2004; Nolen-Hoeksema, 1987, 1991), and have been used in prior research to assess rumination during challenging social interactions (Low et al., 2019).

Emotional suppression. Participants rated three items: "I tried to control or suppress my negative emotions", I tried to hide my negative thoughts and feelings", "I kept my negative emotions to myself' $(1=$ Not at all, $7=$ Very much; $\alpha=.79)$. These items were derived from the Emotion Regulation Questionnaire (ERQ; Gross \& John, 2003) and have been used in many prior studies to assess emotional suppression during social interactions and daily life (e.g., Cameron \& Overall, 2018; Low et al., 2017, 2019; Peters et al., 2019).

Cognitive reappraisal. Two items adapted from the ERQ (Gross \& John, 2003) assessed cognitive reappraisal: "I tried to change the way I was thinking to feel better about the situation", "I tried to think in a way that helped me stay calm" $(1=$ Not at all, $7=$ Very much; $\alpha=.76)$. These items have been used in prior research to assess cognitive reappraisal during social interactions and weekly life (Low et al., 2017, 2019).

\section{Alternative Explanation Measures Assessed During Lockdown}

We gathered additional measures to rule out the possibility that any effects of emotion regulation strategies were due to the challenges of the pandemic, including general 
difficulties with controlling emotions, levels of life stress, and perceived support. The items for each measure were averaged.

Emotion control difficulties. Participants rated three items derived from the Difficulties with Emotion Regulation Scale (DERS; Gratz \& Roemer, 2004): "I felt like my emotions were out of control", "I had difficulty managing my emotions", "I felt like I couldn't control my behavior" $(1=$ Not at all, $7=$ Very much; $\alpha=.89)$.

Stress. Participants rated 12 items to assess how stressful an array of central life domains were during the lockdown (see Neff \& Broady, 2011), such as "parenthood", "living conditions", "your health" ( $1=$ Not at all stressful, $7=$ Extremely stressful; $\alpha=.86)$.

Perceived support. Participants rated seven items assessing how much support they perceived from their partner during the lockdown, such as how often their partner had "given you support", "offered you encouragement" $(1=$ Never, $5=$ Always; $\alpha=.92)$.

\section{Results}

Table 1 presents descriptive statistics. Pre-lockdown and lockdown assessments of emotional well-being, energy/fatigue and general health are not directly comparable because the pre-lockdown assessments asked participants to reflect on their health across the prior four weeks, whereas the lockdown assessments were worded specific to the lockdown period. However, both pre-lockdown and lockdown assessments of depressive symptoms asked participants to rate the presence of symptoms according to frequency per week. Average levels of depressive symptoms increased from pre-lockdown $(M=6.34, S D=4.52)$ to lockdown $(M=7.62, S D=5.10 ; t(364)=-4.25, p<.001)$, and calculating the reliable change index (Jacobson \& Truax, 1991) revealed that 47 out of 365 participants (13\%) demonstrated a clinically significant within-person change in depressive symptoms. Nonetheless, regardless of absolute levels across variables and time points, our analyses controlling for pre-lockdown levels of psychological and physical health provide evidence that any significant effects of 
emotion regulation strategies on psychological and physical health during lockdown reflect relative residual changes from general pre-lockdown health.

Table 2 presents correlations across all measures. The positive correlations between the three emotion regulation strategies are similar to other investigations assessing emotion regulation within specific contexts (e.g., use during specific days, weeks, or emotionally relevant situations) indicating that, when people experience emotional or stressful situations, they typically employ a range of strategies to regulate emotions (Brockman et al., 2016; Ford et al., 2019; Low et al., 2017, 2019). As detailed in the OSM, confirmatory factor analyses supported the distinction between the three emotion regulation measures, and variance inflation factor (VIF) and tolerance statistics indicate that multicollinearity was not an issue in models in which all emotion regulation strategies (and other control variables, such as emotion control difficulties) were modelled simultaneously. Not surprisingly, depressive symptoms and emotional well-being were highly correlated, and these indicators were also strongly associated with energy (low fatigue; see Table 2). We analyze each measure separately given the pre-lockdown distinctions across assessments, the scoring system associated with the RAND-36 Health Survey, and to ensure readers are able to interpret the effects in line with how the scales are typically used in the wider literature.

\section{Primary Analyses: Emotion Regulation and Psychological and Physical Health}

All three emotion regulation strategies were significantly associated with psychological and physical health outcomes in the expected directions (see Table 2). Our primary analyses examine whether each emotion regulation strategy was associated with residual changes in psychological and physical health by controlling for baseline (prelockdown) assessments of psychological and physical health. Given 314 participants were drawn from the same family, we followed the guidelines by Kenny et al. (2006) using the MIXED procedure in SPSS 27 to run dyadic regression models that accounted for the dyadic dependencies in the data (see OSM for annotated syntax and further information). 
We first ran separate analyses to assess the associations between each emotion regulation strategy and each health outcome by regressing each measure of psychological/physical health during lockdown on the (1) pre-lockdown assessment of psychological/physical health so that any significant effects represented the degree to which emotion regulation during the lockdown was associated with relative residual changes in psychological/physical health, and (2) each emotion regulation strategy (in separate models). As shown in the top of Table 3, and as expected, greater rumination and emotional suppression were associated with greater depressive symptoms, and lower emotional wellbeing, energy and general health, accounting for pre-pandemic levels of health.

Cognitive reappraisal — a proposed adaptive emotion regulation strategy—also was associated with poorer health, likely because of the positive correlations across strategies (see Table 2) indicating that the more people needed to regulate emotions during a challenging situation the more they enacted a range of emotion regulation strategies (also see Kivity et al., 2016; Low et al., 2017). Accordingly, following prior research (e.g., Low et al., 2019) to examine the unique effects of emotion regulation strategies accounting for these shared associations and the general need to regulate emotions, we ran models including the three emotion regulation strategies as simultaneous predictors to calculate the unique associations between rumination, emotional suppression, and cognitive reappraisal, with each health outcome. As shown in the bottom of Table 3, greater rumination was uniquely associated with greater depressive symptom, poorer emotional well-being and lower energy (greater fatigue), and greater emotional suppression was uniquely associated with greater depressive symptoms and poorer emotional well-being. By contrast, greater cognitive reappraisal only was independently associated with lower energy (greater fatigue), perhaps because of the energy required to engage in cognitive reappraisal. 


\section{Additional Analyses: Ruling out Alternative Explanations}

All three emotion regulation strategies were associated with greater difficulties in controlling emotions and experiencing more stress (see Table 2), which provide additional evidence that individuals facing greater challenges use a range of strategies to try to regulate their negative emotions. To provide further evidence that the results were not explained by the emotional challenges of the pandemic, we present additional analyses modelling the effects of each emotion regulation strategy simultaneously (as shown by the models in the bottom of Table 3) controlling for (in separate analyses) (1) emotion control difficulties (an indicator that people were having difficulty coping with their emotions), (2) stress (an index of the challenging nature of the lockdown), and (3) perceived support (assessing how much the challenge of the lockdown was potentially eased by partners).

Given that none of the emotion regulation strategies were uniquely associated with perceptions of general health in the primary analyses (see bottom of Table 3), we ran these additional analyses only examining the effects on depressive symptom, emotional well-being and energy (low fatigue). As shown in Table 4, rumination continued to be significantly associated with greater depressive symptoms, poorer emotional well-being and energy controlling for the shared associations across emotion regulation strategies and the significant effects of emotion control difficulties, stress and perceived support. Emotional suppression also continued to be significantly associated with greater depressive symptoms and poorer emotional well-being across these control analyses. Finally, however, the unexpected link between cognitive reappraisal and lower energy (greater fatigue) was reduced when controlling for stress suggesting that this link may be the result of enacting cognitive reappraisal to deal with more stressful situations.

\section{Study 1 Discussion}

Study 1 provided initial evidence that different emotion regulation strategies during a COVID-19 lockdown were associated with residual changes in psychological health and 
energy, but not general perceptions of physical health. Accounting for pre-pandemic psychological health, greater rumination and emotional suppression were associated with greater depressive symptoms and lower emotional well-being, and these effects were robust when controlling for the need to enact emotion regulation (shared associations across emotion regulation strategies) and when controlling for the emotional challenges of the pandemic as indexed by emotion control difficulties, stress, or perceived support. Greater rumination was also robustly associated with lower energy (greater fatigue) during the lockdown, but the effects of cognitive reappraisal on lower energy (greater fatigue) appeared to be explained by greater stress across life domains.

\section{Study 2}

In Study 2, we drew on a larger study from a nationally representative panel studythe New Zealand Attitudes and Values Study (NZAVS) — that included similar measures as Study 1, including psychological and physical health before the pandemic and during the nationwide lockdown as well as items assessing rumination, emotional suppression, and cognitive reappraisal. Our aim was to replicate the effects of Study 1 in a larger, broader sample that allowed us to account for various demographic factors, increasing generalizability. In addition, because participants in the NZAVS are sampled at the same time each year, the time between pre-pandemic and lockdown assessments was more uniform than Study 1, which varied because participants entered the initial pre-pandemic study at different time points. As in Study 1, our primary analyses examined whether the emotion regulation strategies reported at the time of lockdown were associated with relative levels of psychological and physical health accounting for psychological and physical health the previous year. Like Study 1, we also tested the degree to which the links between emotion regulation and psychological and physical health were independent of emotion control difficulties and perceived support. Additionally, in Study 2 we also had pre-pandemic measures of emotion regulation, which we examined to test the theorized importance of 
emotion regulation during challenging situations (i.e., at the time of the lockdown) for psychological and physical health rather than the associations simply arising from stability in emotion regulation and health across time points.

\section{Method}

\section{Participants}

The NZAVS is an ongoing 20-year national longitudinal panel study of social attitudes, personality, and health outcomes that began in 2009. Over the first decade of the NZAVS, samples were drawn from random electoral roll samples, stratified electoral roll samples, and opt-ins through word-of-mouth (see Sibley, 2020 for a detailed description of the sampling procedure for the NZAVS). The eleventh wave of the study commenced in October 2019 and continued during the nationwide Level 4 lockdown. The sample analyzed in the current study involve participants who completed the survey during the nationwide Level 4 lockdown (25 March - 27 April 2020) as well as around the same time period the prior year in the tenth wave of the study. Sibley et al. (2020) reported the mean-level differences in pre-pandemic and lockdown psychological and physical health for participants who completed the survey in the first 18 days of the Level 4 lockdown ( $N S=918-940$ across analyses). We extend this initial examination in the current study by analyzing all data gathered across the entire Level 4 lockdown period (matching the focus in Study 1) and examine the associations between emotion regulation and residual changes in psychological and physical health. The sample of participants with pre-pandemic and lockdown measures for the analyses included 1,607 individuals $(N \mathrm{~s}=1,595-1607$ across primary analyses due to missing data on some health outcomes). See Table 5 for demographics.

\section{Procedure and Materials}

The NZAVS is reviewed every three years by the University of Auckland Human Participants Ethics Committee (approval number 014889 for the waves analyzed here). At both pre-pandemic and lockdown assessments, the NZAVS survey included measures of 
psychological health (psychological distress, personal well-being), physical health (fatigue [low energy], general health), emotion regulation (rumination, emotional suppression, cognitive reappraisal) and two of the control variables used in Study 1 (emotion control difficulties, perceived support). Details about all measures are available here. The data used in the research cannot be publicly shared as the data are part of a large ongoing longitudinal study with stringent privacy rules. The syntax and Mplus output are available here: https://osf.io/e5ftj/?view_only=eeccf5b10c1d486782ef0c278443e4d0.

\section{Psychological and Physical Health}

The measures are labelled and scored as they were intended for use in the NZAVS, which means some labels are not equivalent with Study 1 despite assessing similar constructs (e.g., psychological distress vs. depressive symptoms), some measures are scored differently despite assessing the same measure (e.g., fatigue [low energy] vs energy [low fatigue]), and some measures are broader (personal well-being across domains vs. emotional well-being). These distinctions also mean that the associations across health measures were not as strong in Study 2 compared to Study 1 (see Table 7), and thus the tests in Study 2 may provide a stronger test of the breadth of effects of emotion regulation during lockdown.

Psychological distress. The NZAVS includes the Kessler-6, a short-form scale of psychological distress (Kessler et al., 2010) that includes 6 items that overlap with the CES-D items used to assess depressive symptoms in Study 1: "During the last 30 days, how often did.... you feel hopeless?”; “you feel so depressed that nothing could cheer you up?”; "you feel that everything was an effort?”; “you feel worthless?”; “you feel restless or fidgety?”; "you feel nervous?" $(0=$ None of the time, $4=$ All of the time; Pre-Lockdown $\alpha=.85$, Lockdown $\alpha=.86)$.

Personal Well-being. The NZAVS assessment of well-being represents a more global measure than the emotional well-being assessed in Study 1. Participants rated how satisfied they were with their "standard of living," "health," "future security," and "personal 
relationships" ( $0=$ completely dissatisfied, $10=$ completely satisfied; Cummins et al., 2003; Pre-Lockdown $\alpha=.73$, Lockdown $\alpha=.73$ )

Fatigue (Low Energy). The NZAVS includes one item assessing fatigue: "During the last 30 days, how often did.... you feel exhausted?" $(0=$ None of the time, $4=$ All of the time $)$.

General Health. The NZAVS uses the short-form general physical health scale from the SF-36 used in Study 1, including three items: "In general, would you say your health is... $(1=$ Poor, $5=$ Excellent $)$, and "I seem to get sick a little easier than most people", "I expect my health to get worse" (reversed-coded; 1 = Definitely false, 5 = Definitely true; PreLockdown $\alpha=.58$, Lockdown $\alpha=.62$ ).

\section{Emotion Regulation}

Rumination was assessed with a single item adapted from Nolen-Hoeksema and Morrow (1993): "During the last 30 days, how often did.... you have negative thoughts that repeated over and over?" $(0=$ None of the time, $4=$ All of the time $)$.

Emotional suppression and cognitive reappraisal were each assessed with single items similar to those used in Study 1: "When I feel negative emotions, I suppress or hide my emotions" and "When I feel negative emotions, I change the way I think to help me stay calm" ( 1 = strongly disagree, $7=$ strongly agree $)$. Unlike the assessment of rumination or the measures in Study 1, these items did not specifically assess emotion regulation within the lockdown period. We expected that current experiences would be salient and so that participants' ratings completed during the lockdown period would capture variation in emotion regulation during the lockdown (in addition to potentially incorporating more general tendencies). This assumption is supported by the associations across pre-pandemic and lockdown assessments of emotional suppression $(r=.49)$ and cognitive reappraisal $(r=$ .41; see Table 7), which indicate use of these strategies were not extremely stable across time. 


\section{Alternative Explanation Measures}

Emotion control difficulties were assessed with a single item similar to the items used in Study 1: "When I feel negative emotions, my emotions feel out of control" $(1=$ strongly disagree, 7 = strongly agree).

Perceived support was assessed with three items adapted from Cutrona and Russell (1987): “There are people I can depend on to help me if I really need it", "There is no one I can turn to for guidance in times of stress" (reverse-coded), and "I know there are people I can turn to when I need help" ( 1 = very inaccurate, 7 = very accurate; Pre-Lockdown $\alpha=$ .80 , Lockdown $\alpha=.82$ ).

\section{Demographic Covariates}

Gender was assessed using an open-ended item and coded according to the NZAVS gender coding scheme $(0=$ women, $1=$ men; Fraser et al., 2019). Participants self-reported date of birth was used to calculate their age. Ethnicity was assessed with the standard Statistics New Zealand (2020) question which allows for multiple ethnic identities. Analyses included codes for the minority groups "Māori" $(0=$ no, $1=$ yes $)$, Pasifika $(0=$ no, $1=$ yes $)$, and Asian $(0=$ no, $1=$ yes $)$ with the reference group representing the NZ Pākehā /European majority. We used two indicators of socio-economic status, the New Zealand SocioEconomic Index (Fahy et al., 2017) and the New Zealand Deprivation Index (Atkinson et al., 2014). Participants' highest level of education was asked with an open-ended question and then coded according to levels of education recognized by the New Zealand Qualifications Authority (2012), leaving a 0 (low) to 10 (high scale). Binary (yes/no) variables were created from open-ended or multi-response items to indicate whether participants were: employed, in a serious relationship, a parent, lived in an urban area, and were born in New Zealand or not. See Table 5. 


\section{Results}

Table 6 presents descriptive statistics. In general, average levels of psychological and physical health and emotion regulation did not differ across pre-lockdown to lockdown assessments, with the exception that people reported lower fatigue (also see Sibley et al., 2020) and greater cognitive reappraisal. Calculating the reliable change index (Jacobson \& Truax, 1991) as in Study 1 revealed that 129 out of 1,595 participants (8\%) demonstrated a clinically significant change in psychological distress from pre-lockdown to lockdown. In the context of this relative resilience on average, our analyses tested whether the use of different emotion regulation strategies during the lockdown were associated with relative levels of psychological and physical health accounting for pre-pandemic levels of health.

\section{Primary Analyses: Emotion Regulation and Psychological and Physical Health}

As shown in Table 7, emotion regulation strategies were associated with psychological and physical health prior to the lockdown (above the diagonal) and during the lockdown (below the diagonal). To assess whether the use of each emotion regulation strategy during the lockdown was associated with psychological and physical health accounting for psychological and physical health prior to the pandemic, our primary analyses mimicked that of Study 1 with two exceptions. First, as evident in Table 7, the associations between the three emotion regulation strategies were not as strong as in Study 1, probably because the assessments in Study 2 were not worded to assess emotion regulation within the specific context of the lockdown. Given these distinctions and combined with the larger sample and established links in Study 1, our initial models regressed psychological and physical health during lockdown on all three emotion regulation strategies simultaneously to calculate the unique associations between rumination, emotional suppression, and cognitive reappraisal with psychological and physical health during lockdown (controlling for prelockdown psychological and physical health). 
Second, given the larger and more diverse sample, and following procedures applied across analyses using NZAVS data, we included in all regression models a standard set of demographic covariates, including gender, age, ethnicity, socioeconomic status, education attainment, employment, relationship and parent status, urban residence, and born in NZ; see Table 5). For clarity of presentation, we present the effects of emotion regulation in Table 8 and present the effects of the demographic covariates in the OSM (see Table SM7). In general, the demographic variables did not predict variation in outcomes although older participants reported lower depressive symptoms and fatigue, people in close relationships reported higher personal well-being, and greater deprivation was associated with poorer general health. These demographic differences were controlled for across analyses.

The results in Table 8 show a clear, strong pattern of independent associations between emotion regulation during the lockdown and psychological and physical health. Both greater rumination and emotional suppression during the lockdown were uniquely associated with greater psychological distress and fatigue (low energy) as well as lower personal wellbeing and general health accounting for pre-pandemic levels of these variables. In contrast, and unlike Study 1, cognitive reappraisal during the lockdown was unrelated to fatigue (low energy) but was significantly associated with lower psychological distress as well as greater personal well-being and general health accounting for pre-pandemic levels of these variables.

\section{Additional Analyses: Ruling out Alternative Explanations}

As in Study 1, rumination and emotional suppression were associated with greater difficulties in controlling emotions (and, unlike Study 1, cognitive reappraisal was associated with lower difficulties in controlling emotions; see Table 7). As in Study 1, to illustrate that the results were not explained by the emotional challenges of the pandemic, we present additional analyses controlling for (1) emotion control difficulties (and in separate analyses)

(2) perceived support. As displayed in Table 9, the significant associations between rumination during lockdown and all four health outcomes remained when controlling for 
emotion control difficulties and perceived support. Emotional suppression also continued to be significantly associated with all health outcomes, except for personal well-being when controlling for the strong links between perceived support and personal well-being. Finally, the positive association between cognitive reappraisal during the lockdown and personal well-being and general health remained, but the links between cognitive reappraisal and psychological distress were reduced when controlling for emotion control difficulties ( $p=$ $.030)$ and perceived support $(p=.054)$.

\section{Additional Analyses Examining Emotion Regulation Prior to the Pandemic}

Our primary aim was to examine how emotion regulation enacted during the challenging context of the lockdown was associated with variation in relative levels of psychological and physical health accounting for pre-pandemic health outcomes. However, the longitudinal design of NZAVS allowed us to also test the degree to which emotion regulation assessed during the lockdown was associated with residual changes in psychological/physical health controlling for the stable associations between emotion regulation and health prior to the pandemic (see Table 7). We expected that emotion regulation during lockdown would continue to be associated with residual changes in psychological/physical health irrespective of emotion regulation reported 1-year earlier, and that any effects of pre-lockdown emotion regulation would be the result of the current enactment of emotion regulation during the challenging situation of the lockdown.

We first ran analyses examining the effects of emotion regulation assessed prior to the pandemic on psychological/physical health during the lockdown (accounting for prepandemic health) by rerunning the primary analyses in Table 8 replacing lockdown emotion regulation strategies with pre-pandemic assessments of emotion regulation strategies. We present these effects in the OSM (see Table SM8). Rumination assessed prior to the pandemic significantly predicted poorer health outcomes during the lockdown, including greater psychological distress, lower personal well-being, greater fatigue, and poorer general 
health. Greater pre-pandemic emotional suppression also predicted greater psychological distress and fatigue during lockdown accounting for pre-pandemic health. There were no significant effects of pre-pandemic cognitive reappraisal on health during the lockdown.

We next reran our primary analyses in Table 8 assessing the associations between emotion regulation during lockdown and psychological/physical health adding in each of the emotion regulation strategies assessed prior to the pandemic. As expected, when including all three emotion regulation strategies assessed at both time points, all of the associations between the emotion regulation strategies assessed during lockdown and health outcomes remained significant, whereas the effects of emotion regulation assessed prior to the lockdown described above were no longer significant (see OSM, Table SM9). These analyses illustrate that the links between emotion regulation during lockdown and psychological and physical health arise from the specific emotion regulation strategies assessed at the time of the lockdown and were independent of the stable associations between emotion regulation and psychological and physical health across time points.

\section{Study 2 Discussion}

The results of Study 2 replicated and extended the findings from Study 1. As in Study 1, greater rumination and emotional suppression during the lockdown were associated with poorer psychological health (accounting for pre-pandemic health). In contrast to Study 1, rumination and emotional suppression were both also independently associated with poorer physical health, including greater fatigue and poorer perceptions of general health. Moreover, unlike Study 1, the results in Study 2 provided evidence that cognitive reappraisal has protective effects, including positive associations with personal well-being and general health (accounting for pre-pandemic well-being and health). Differences in the measures and design across studies may explain why the associations between emotion regulation and health were generally stronger across health outcomes in Study 2. More distinct associations across the measures of emotion regulation and health, more uniform (1-year) time period between pre- 
pandemic and lockdown assessments, and the larger and more diverse sample likely enhanced the reliability and the power of the tests in Study 2. In addition, the measure of cognitive reappraisal in Study 2 specifically referred to reappraising when experiencing negative emotions (as the items for rumination and suppression did in both studies) and so may have more precisely assessed the importance of this strategy to manage emotional challenges. We consider the relevance and meaning of the differences across studies as we reflect on the implications and contributions of the current results in the General Discussion.

\section{General Discussion}

Can affective science be applied to understand who might be most at risk for poor psychological and physical health versus who remains resilient during the COVID-19 pandemic? Not surprisingly, there are major concerns that the pandemic will negatively affect psychological and physical health as people across the globe face ongoing economic, social and personal challenges. Such challenges may be particularly acute during COVID-19 lockdowns in which people are confronted with increased work and family demands while being isolated from support networks. Yet, many people show resilience in the face of substantial demands and relative levels of psychological and physical health are likely to be shaped by the strategies people use to regulate their emotions during this unprecedented time.

The results of two studies that assessed psychological and physical health prior to and during the pandemic provide support that the use of different emotion regulation strategies during the challenges of the pandemic have important implications for sustaining health and well-being. Study 1 leveraged an ongoing study in which parents had completed assessments of their psychological and physical health prior to the pandemic. Parents $(N=365)$ then completed assessments of emotion regulation and psychological and physical health when they were confined to the home with their young children during a mandated nationwide COVID-19 lockdown. Study 2 leveraged a nationally representative longitudinal panel study to draw upon a sample of adults $(N=1,607)$ who had completed assessments of both emotion 
regulation and psychological and physical health a year prior to completing the same assessments during the mandated nationwide COVID-19 lockdown.

Despite warnings that the pandemic poses considerable risk to health and well-being, the results across both studies indicated that, in general, participants in the current studies were relatively resilient. Nonetheless, the results also demonstrated that, accounting for prepandemic levels of psychological and physical health, the use of different emotion regulation strategies were reliably associated with relative levels of psychological and physical health during the lockdown. In both studies, greater rumination and emotional suppression were associated with poorer psychological health (greater depressive symptoms and psychological distress, lower emotional and personal well-being), even when controlling for the emotional challenges of the pandemic (emotion control difficulties, life stress, perceived support) as well as a range of demographic covariates. Greater rumination was also associated with greater fatigue (lower energy) in both studies. Additional associations also emerged in Study 2, which included a larger and more diverse sample. Rumination and emotional suppression were related to poorer general health, and although cognitive reappraisal showed few unique associations in Study 1, cognitive reappraisal was positively associated with personal wellbeing and general health in Study 2 (controlling for pre-pandemic well-being and health).

By assessing psychological and physical health prior to the pandemic and during a mandatory nationwide lockdown, these studies illustrate that the effects of emotion regulation most often demonstrated in experimental studies, or within the context of daily life and social interactions, emerge in more extreme situations when emotion regulation matters most. In doing so, the results provide evidence that key models in affective science provide insight into health risk versus resilience within a real-world emergent context. Moreover, as discussed below, examining the unique associations of different emotion regulation strategies during lockdown with psychological and physical health, as well as ruling alternative 
explanations within this distinct context, advances understanding of the connections and distinctions across different emotion regulation strategies.

\section{Rumination and Emotional Suppression are Independent Vulnerabilities for Poorer Psychological and Physical Health During the Pandemic}

The current research provides novel evidence that different emotion regulation strategies have important implications for psychological health during the COVID-19 pandemic. Accounting for psychological health assessed prior to the pandemic, emotion regulation strategies typically considered to be "maladaptive", including rumination and emotional suppression, were independently associated with poorer psychological health, including greater depressive symptoms and psychological distress as well as decreases in well-being during a nationwide lockdown. These results are consistent with the central role rumination is theorized to play in the development and maintenance of depression (NolenHoeksema, 2000; Spasojevic et al., 2004), but demonstrate the importance of rumination in a context in which rumination should have significant consequences. The results also establish that the associations between rumination and psychological/physical health are unique from emotional suppression and cognitive reappraisal, and were not accounted for by the relative emotional challenges of the situation (as indexed by difficulties in controlling emotions, stress across life domains, and perceived support). Similarly, emotional suppression has been reliably associated with poorer well-being (Gross, 2015; Webb et al., 2012), but the unique effects of emotional suppression compared to other maladaptive emotion regulation strategies have rarely been tested nor have the independent associations between suppression and psychological/physical health been tested in crisis situations.

The pattern of results testing the independent associations between rumination and emotional suppression with psychological and physical health highlights similarities and differences in the way these strategies may shape health outcomes. On the one hand, a range of similar processes likely explain why rumination and emotional suppression represent a 
vulnerability for health and well-being. Both rumination and emotional suppression can interfere with self-regulation and deplete cognitive resources, which make it difficult for individuals to engage in problem solving (e.g., Clore \& Gasper, 2000; Franchow \& Suchy, 2015; Watkins \& Baracaia, 2002). These types of internal mechanisms may be particularly influential during COVID-19 lockdowns compared to other social mechanisms given that everyone was facing isolation from others outside the home. Indeed, although both rumination and emotional suppression can interfere with the constructive expression of emotions that elicit support from others (e.g., Nolen-Hoeksema \& Davis, 1999; Srivastava et al., 2009), both strategies were only weakly associated with lower support. Moreover, rumination and emotional suppression continued to be associated with poorer psychological health when controlling for perceived support indicating that support was not a strong explanatory (i.e., mediating) factor, with the exception that emotional suppression was no longer associated with personal well-being in Study 2 when controlling for perceptions of support. Thus, the social nature of suppressing emotions from others may mean that, compared to rumination, social processes play a greater explanatory role in the links between emotional suppression and health outcomes (e.g., English \& John, 2013; Srivastava et al., 2009), but the social confinement of the lockdowns may have reduced the degree to which this social pathway emerged in the current studies.

Rumination might also have additional effects beyond emotional suppression that accounted for the more consistent, robust associations between rumination and poorer psychological and physical health. Indeed, comparison of the effect sizes in both studies (see Tables 3 and 8) reveal that the independent effects of rumination on psychological health were 3-4 times larger than the effects of emotional suppression. Moreover, rumination was robustly associated with greater fatigue in both studies along with poorer physical health in Study 2. By contrast, emotional suppression was only associated with greater fatigue and poorer general health in Study 2 and these effects were half the size of rumination (see Table 
8). This relative pattern may indicate that rumination poses a stronger risk factor for poorer health and well-being in this context, perhaps because the unpredictable and uncontrollable nature of the pandemic may amplify the tendency to repeatedly ruminate on the situation and, in turn, exacerbate resulting depletion and distress. Indeed, prior research indicates intolerance of uncertainty predicts greater depressive symptoms because such intolerance amplifies rumination (de Jong-Meyer et al., 2009; Yook et al., 2010). Yet, it is also possible that the larger effect sizes of rumination arise because measures of rumination and depressive symptoms strongly overlap. As is common (Olatunji et al., 2013), rumination and psychological health were highly correlated within each time point. Thus, despite theoretical models and empirical evidence differentiating rumination and depressive symptoms, it is possible that rumination is so reciprocally bound to depressed mood that the strong effects of rumination simply represent the continued presence of a broader, overarching syndrome.

Nonetheless, the consistent effects of both rumination and emotional suppression found in the current studies highlight the importance of teasing apart the relative contributions of different emotion regulation strategies for psychological and physical health, especially within challenging contexts such as COVID-19 lockdowns.

\section{Mixed Evidence that Cognitive Reappraisal Facilitates Psychological and Physical}

\section{Health During the Pandemic}

Cognitive reappraisal had mixed effects across studies showing independent, robust associations with only three (out of eight) outcomes tested across Studies 1 and 2. In Study 1, greater use of cognitive reappraisal was robustly associated with lower energy (greater fatigue), but not uniquely associated with any other outcomes after controlling for the need to use emotion regulation strategies (use of rumination and emotional suppression) and the emotional challenges of the lockdown (emotion control difficulties, life stress, perceived support). In Study 2, however, greater cognitive reappraisal was not associated with fatigue, but was robustly associated with better personal well-being and general health. The mixed 
findings of cognitive reappraisal are somewhat at odds with prior evidence showing that cognitive reappraisal is generally associated with better psychological functioning and wellbeing (e.g., Gross \& John, 2003; Kim et al., 2021), although consistent with other research indicating that cognitive reappraisal may not have unique effects beyond rumination or emotional suppression within stressful social interactions (e.g., Low et al., 2017, 2019).

One possibility for the lack of associations shown in Study 1 and some prior studies is that the items assessing rumination and emotional suppression referred specifically to regulating negative emotions and thus were more relevant to the emotional challenges of the pandemic. Yet, the cognitive reappraisal items assessed the degree to which people attempted to think in ways that helped them feel better about the situation and stay calm, which similarly captured a need to regulate negative or stressful states in order to feel better or stay calm. Moreover, cognitive reappraisal was positively associated with rumination and emotional suppression in Study 1, which involved assessments of the specific use of emotion regulation strategies during the lockdown. These positive associations are consistent with people needing to employ a range of emotion regulation strategies in specific challenging contexts (e.g., Brockman et al., 2016; Ford et al., 2019; Low et al., 2017, 2019), and inconsistent with the possibility that cognitive reappraisal measures capture positive rather than negative emotional states (which would yield negative or weaker associations with rumination and suppression). Finally, in Study 2, the assessment of cognitive reappraisal specifically referred to the enactment of this strategy when experiencing negative emotions. In this larger sample, cognitive reappraisal had robust positive associations with two (personal well-being, general health) of four outcomes, and these protective effects cannot be accounted for by a focus on positive emotions given all measures referred to the regulation of negative emotions.

Furthermore, both studies provided evidence that cognitive reappraisal was as, or even more, relevant to the challenging context of the lockdown. Average levels and variation 
of cognitive reappraisal was as high or higher than the other emotion regulation strategies in both Studies 1 and 2. Moreover, in Study 2, which included assessments of emotion regulation prior to the pandemic and during the lockdown, mean-level comparisons revealed that, on average, participants reported increases in the use of cognitive reappraisal. This novel finding indicates a general tendency for greater enactment of cognitive reappraisal to deal with the challenges of the pandemic and lockdowns. Although the results differed across studies, the increases in cognitive reappraisal shown in Study 2 may help explain why greater cognitive reappraisal was associated with greater fatigue in Study 1; parents may have required more energy and resources to use this strategy given the uncontrollable stressors they were encountering during lockdown. Moreover, although the more powerful analyses in Study 2 suggested that greater cognitive reappraisal helped sustain well-being and general health during this time, cognitive reappraisal did not have beneficial effects on fatigue. Nonetheless, it may be important for future research to assess whether the implied valence in assessments of emotion regulation play a role in the differential effects of different strategies, and whether the potential benefits of cognitive reappraisal may vary across different contexts.

Indeed, the relative success and potential opposing effects of cognitive reappraisal may help explain the mixed results for cognitive reappraisal. Prior research suggests that greater cognitive reappraisal ability is particularly important for psychological health when facing situations of high uncontrollable stress when only emotional reactions to the situation — and not the situation itself — can be changed (Troy et al., 2013). Yet, these benefits likely only occur if people's reappraisal attempts are successful. The results of Study 2 suggested a trend for increases in cognitive reappraisal attempts to traverse the challenges of the pandemic, but prior experimental and diary studies show that many individuals who attempt cognitive reappraisal are unable to reframe the situation (Ford et al., 2017; Troy et al., 2010). The high levels of uncontrollability, uncertainty and unpredictability of the pandemic and lockdowns may make it very difficult to establish and sustain concrete, 
positive and meaningful reappraisals even for people who have high cognitive reappraisal ability. This difficulty, and associated need for repeat reappraisal attempts, may have a range of costs (e.g., depletion and fatigue) that dilutes the potential protective effects of cognitive reappraisal. Moreover, recent evidence during the pandemic also highlights that cognitive reappraisal can have opposing effects, such as reducing fear and sustaining well-being while also reducing the enactment of behaviors required to protect physical health (Smith et al., 2021). Thus, cognitive reappraisal may show more beneficial effects for people who are able to view the situation in ways that facilitate equanimity without depleting the regulatory resources they need to address what can actually be done to promote well-being and protect physical health within the confines of the pandemic.

\section{Strengths, Caveats, and Future Research}

Leveraging two ongoing longitudinal studies provided strong tests of whether emotion regulation enacted during a mandated nationwide lockdown played an important role in the relative risk versus resilience of psychological and physical health during the COVID-19 pandemic. Nonetheless, the correlational nature of the data limits causal conclusions and opens the possibility of third variables. Perhaps the most challenging alternative explanation is that the effects of emotion regulation simply capture the additional emotional challenges of the pandemic. When assessing the specific use of emotion regulation within the confines of the lockdown in Study 1, rumination, emotional suppression, and cognitive reappraisal were strongly positively associated (as in other studies assessing emotion regulation in specific emotionally relevant contexts). This shared association provides confirming evidence that the lockdown created a relevant context to investigate the effects of various emotion regulation strategies, but also highlights the importance of controlling for this shared association to rule out that any effects were due to the need to enact a range of emotion regulation strategies. Additional analyses in both studies demonstrated that rumination and emotional suppression had independent associations with important psychological and physical health outcomes (as 
did cognitive reappraisal on two outcomes in Study 2) revealing that it is unlikely that the effects were simply due to facing challenges and needing to regulate emotions.

Further analyses also provided additional evidence that the links between emotion regulation and health were not simply due to the emotional challenges of the pandemic. In Study 1 , the effects of rumination and emotional suppression on psychological health remained when controlling for (1) general emotion control difficulties, which is a good indicator that people were having difficulty coping with the emotional challenges of the pandemic, (2) stress across a number of life domains, which provided an index of the broader challenging nature of the lockdown, and (3) perceived support, which provided an index of how much the emotional challenge of the pandemic was potentially eased by partners. In Study 2, which did not have a distinct measure of life stress, the significant associations between all three emotion regulation strategies and psychological/physical health remained controlling for the shared associations across emotion regulation strategies, emotion control difficulties, and perceived support. These results provide good evidence that the replicated links across studies were not just a symptom of the emotional challenges of the pandemic.

Our aim was to assess the effects of emotion regulation strategies enacted in the midst of a novel challenging situation, and thus we assessed the degree to which emotion regulation during the lockdown were associated with health accounting for pre-pandemic health. Another potential longitudinal design would be to examine how habitual emotion regulation assessed prior to the pandemic predicted psychological and physical health during the lockdown. However, any effects of habitual emotion regulation on context-specific outcomes should be (in large part) the result of the use of emotion regulation strategies within that context, and thus any effects of pre-pandemic emotion regulation should be mediated by the particular use of emotion regulation within that context (see Low et al., 2017; Peters et al., 2020). The results of Study 2 supported our theorizing. Greater rumination and emotional suppression assessed 1-year prior to the lockdown predicted poorer psychological and 
physical health during the lockdown (accounting for pre-pandemic health), but these effects were accounted for by the enactment of rumination and suppression during the lockdown. Thus, the results indicate that the ways people attempted to regulate emotions within the lockdown context played an important role in their relative resilience independent of the stable associations between emotion regulation and health across time.

Although the effects of rumination and emotional suppression on psychological health replicated across Studies 1 and 2, the results for physical health differed across studies. None of the emotion regulation strategies independently predicted general perceptions of physical health in Study 1, but all three strategies were reliably associated with general health in Study 2. The associations between emotion regulation and physical health may be smaller and less reliable for several reasons. Study 1 involved a smaller and more uniform sample of parents with young children whose general health may be relatively robust, especially at the initial stages of the pandemic. Indeed, timing of assessment in both studies may offer conservative tests of the potential physical health effects. For example, the challenges of the lockdown in the early stages of the pandemic may have been counterbalanced by people having more time to sleep, exercise, spend time with family and participate in leisure activities, which likely accounts for the average decreases in fatigue observed in Study 2 (also see Sibley et al., 2020). The effects on physical (and psychological) health may emerge more strongly over time as people have endured ongoing challenges arising from the pandemic. Finally, it is plausible that emotion regulation more powerfully and immediately impacts psychological health, which in turn undermines physical health across time. A valuable direction for future investigations examining changes across the pandemic will be to examine whether poorer psychological health predicts greater risk of physical health problems across time.

The links between emotion regulation and relative levels of health emerged in the context of relative resilience. For example, focusing on the measures of depressive symptoms and psychological distress that were directly comparable prior to versus during the lockdown, 
the majority of participants did not show marked declines in psychological health. In Study 1, depressive symptoms increased on average across the sample, consistent with other research suggesting that the current crisis risks declines in psychological health. Yet, only $13 \%$ of participants demonstrated a clinically significant change in depressive symptoms. In Study 2, there were no differences in average levels of pre-lockdown versus lockdown psychological distress, and only $8 \%$ demonstrated a clinically significant change in psychological distress. This overall picture of resilience aligns with prior examinations of the effects of disasters and loss, which show that the majority of people, despite facing significant challenges, exhibit sustained health and well-being (e.g., Bonnano et al., 2007, 2008; see Chen \& Bonanno, 2020). The results are also consistent with a review of studies documenting the impact of the COVID-19 pandemic, with average increases in psychological distress accompanied by resilience in life satisfaction and social well-being (Aknin et al., in press).

Such resilience may be more common within samples in which pre-pandemic levels of depressive symptoms and psychological distress were low, and levels of well-being and physical health were high, as to be expected in non-clinical community samples. The risk of clinically significant declines in psychological and physical health are likely stronger for people entering the pandemic with poorer health or who endure greater economic hardship and social isolation. The relative risks to psychological and physical health also may emerge more strongly over time as people across the globe have to continually endure a range of challenges caused by the ongoing pandemic. Yet, regardless of average and absolute levels of psychological and physical health, the current studies provide valuable evidence that the use of different emotion regulation strategies during the lockdown is associated with variation in relative levels of psychological and physical health accounting for pre-pandemic health. Moreover, although our samples and results cannot speak to the risk of developing major depression or disease, even minor or subthreshold depression has a substantial impact on people's daily lives, carries significant economic cost, and risks poorer health and mortality 
outcomes (Cuijpers et al., 2013; Cuijpers et al., 2007; Rucci et al., 2003). Accordingly, the results highlight the potential importance of emotion regulation processes in a context in which emotion regulation should really matter and in samples for which variance in psychological and physical health still has important implications.

\section{Conclusions and Implications}

Inevitable limitations notwithstanding, the findings from the current studies both advance the emotion regulation literature and have important implications for facilitating positive health and resilience during the pandemic (and other crises). First, the current studies provide strong evidence for the utility of key models in affective science in explaining differences in psychological and physical health within the throes of a real-world demanding context. The results indicate that rumination and emotional suppression when facing the challenges of the pandemic may risk poorer psychological and physical health, whereas cognitive reappraisal may be more protective. Second, research examining emotion regulation and health during the pandemic are important because they provide valuable information for the varied ways health and well-being could be supported during and in the aftermath of the pandemic (Gruber et al., 2020). Despite the rapidly growing research into the health and well-being costs of the pandemic, evidence of factors that predict risk versus resilience have been restricted to demographic variables of the type that we controlled for in Study 2 (Aknin et al., in press). Accordingly, there is not enough longitudinal evidence yet to assess the relative effects of emotion regulation compared to other psychological processes that likely play a role in risking versus sustaining psychological and physical health during this time. Our results provide initial evidence that facilitating emotion regulation by helping people sit more comfortably with uncertainty, accept negative emotions, and effectively reappraise emergent challenges may be an important addition to policy, education and therapeutic interventions designed to help sustain health and well-being. 
Footnotes

${ }^{1}$ https://www.bsg.ox.ac.uk/research/research-projects/coronavirus-governmentresponse-tracker 


\section{References}

Ackerman, R.A., Ledermann, T., \& Kenny, D.A. (2016). Power analysis for the ActorPartner Interdependence Model. Retrieved on 23 July, 2020 from https://robertackerman.shinyapps.io/APIMPowerR/

Aknin, L. B., De Neve, J. E., Dunn, E. W., Fancourt, D., Goldberg, E., Helliwell, J., ... Amor, Y. B. (2021, February 19). Mental Health During the First Year of the COVID19 Pandemic: A Review and Recommendations for Moving Forward. https://doi.org/10.31234/osf.io/zw93g

Aldao, A., Nolen-Hoeksema, S., \& Schweizer, S. (2010). Emotion-regulation strategies across psychopathology: A meta-analytic review. Clinical psychology review, 30(2), 217-237. https://doi.org/10.1016/j.cpr.2009.11.004

American Psychological Association. (2020, May). Stress in the time of COVID-19. Retrieved from https://www.apa.org/news/press/releases/stress/2020/stress-in-america covid.pdf

Andresen, E. M., Malmgren, J. A., Carter, W. B., \& Patrick, D. L. (1994). Screening for depression in well older adults: Evaluation of a short form of the CES-D. American journal of preventive medicine, 10(2), 77-84. https://doi.org/10.1016/S0749$3797(18) 30622-6$.

Appleton, A. A., Buka, S. L., Loucks, E. B., Gilman, S. E., \& Kubzansky, L. D. (2013). Divergent associations of adaptive and maladaptive emotion regulation strategies with inflammation. Health Psychology, 32(7), 748-756. https://doi.org/10.1037/a0030068

Atkinson, J., Salmond, C., \& Crampton, P. (2014). NZDep2013 index of deprivation. Wellington: Department of Public Health, University of Otago.

Berman, M. G., Peltier, S., Nee, D. E., Kross, E., Deldin, P. J., \& Jonides, J. (2011). Depression, rumination and the default network. Social cognitive and affective neuroscience, 6(5), 548-555. https://doi.org/10.1093/scan/nsq080 
Bjureberg, J., Ljótsson, B., Tull, M. T., Hedman, E., Sahlin, H., Lundh, L. G., ... \& Gratz, K. L. (2016). Development and validation of a brief version of the difficulties in emotion regulation scale: the DERS-16. Journal of psychopathology and behavioral assessment, 38(2), 284-296. https://doi.org/10.1007/s10862-015-9514-X

Bonanno, G. A., Galea, S., Bucciarelli, A., \& Vlahov, D. (2007). What predicts psychological resilience after disaster? The role of demographics, resources, and life stress. Journal of Consulting and Clinical Psychology, 75(5), 671-682. https://doi.org/10.1037/0022006X.75.5.671

Bonanno, G. A., Ho, S. M. Y., Chan, J. C. K., Kwong, R. S. Y., Cheung, C. K. Y., Wong, C. P. Y., \& Wong, V. C. W. (2008). Psychological resilience and dysfunction among hospitalized survivors of the SARS epidemic in Hong Kong: A latent class approach. Health Psychology, 27(5), 659-667. https://doi.org/10.1037/0278-6133.27.5.659

Bovin M. J., Marx B. P. (2011) The importance of the peritraumatic experience in defining traumatic stress. Psychological bulletin, 137(1), 47-67. https://doi.org/10.1037/a0021353

Bowerman, B. L., \& O'connell, R. T. (1990). Linear statistical models: An applied approach. Brooks/Cole.

Brans, K., Koval, P., Verduyn, P., Lim, Y. L., \& Kuppens, P. (2013). The regulation of negative and positive affect in daily life. Emotion, 13(5), 926939. https://doi.org/10.1037/a0032400

Brockman, R., Ciarrochi, J., Parker, P., \& Kashdan, T. (2017). Emotion regulation strategies in daily life: Mindfulness, cognitive reappraisal and emotion suppression. Cognitive Behaviour Therapy, 46(2), 91-113.

Butler, E. A., Egloff, B., Wilhelm, F. H., Smith, N. C., Erickson, E. A., \& Gross, J. J. (2003). The social consequences of emotional suppression. Emotion, 3, 48-67. http://dx.doi.org/10.1037/1528-3542.3.1.48 
Cameron, L. D., \& Jago, L. (2008). Emotion regulation interventions: A common-sense model approach. British Journal of Health Psychology, 13(2), 215-221. http://dx.doi.org/ 10.1348/135910708X288800

Cameron, L. D., \& Overall, N. C. (2018). Suppression and expression as distinct emotionregulation processes in daily interactions: Longitudinal and meta-analyses. Emotion, 18(4), 465-480. https://doi.org/10.1037/emo0000334

Chen, S., \& Bonanno, G. A. (2020). Psychological adjustment during the global outbreak of COVID-19: A resilience perspective. Psychological Trauma: Theory, Research, Practice, and Policy, 12(S1), S51-S54. http://dx.doi.org/10.1037/tra0000685

Clore, G. L., \& Gasper, K. (2000). Feeling is believing: Some affective influences on belief. In N. H. Frijda, A. S. R. Manstead, \& S. Bem (Eds.), Emotions and beliefs: How feelings influence thoughts (pp. 10-44). Cambridge, UK: Cambridge University Press.

Cludius, B., Mennin, D., \& Ehring, T. (2020). Emotion regulation as a transdiagnostic process. Emotion, 20(1), 37-42. https://doi.org/10.1037/emo0000646

Conway, M., Csank, P. A., Holm, S. L., \& Blake, C. K. (2000). On assessing individual differences in rumination on sadness. Journal of personality assessment, 75(3), 404425. http://dx.doi.org/ 10.1207/S15327752JPA7503_04

Cuijpers, P., Vogelzangs, N., Twisk, J., Kleiboer, A., Li, J., \& Penninx, B. W. (2013). Differential mortality rates in major and subthreshold depression: meta-analysis of studies that measured both. The British journal of psychiatry: the journal of mental science, 202(1), 22-27. https://doi.org/10.1192/bjp.bp.112.112169

Cuijpers, P., Smit, F., Oostenbrink, J., de Graaf, R., Ten Have, M., \& Beekman, A. (2007). Economic costs of minor depression: a population-based study. Acta psychiatrica Scandinavica, 115(3), 229-236. https://doi.org/10.1111/j.1600-0447.2006.00851.x 
Dalgleish, T., Yiend, J., Schweizer, S., \& Dunn, B. D. (2009). Ironic effects of emotion suppression when recounting distressing memories. Emotion, 9(5), 744749. https://doi.org/10.1037/a0017290

de Jong-Meyer, R., Beck, B., \& Riede, K. (2009). Relationships between rumination, worry, intolerance of uncertainty and metacognitive beliefs. Personality and Individual Differences, 46(4), 547-551. http://dx.doi.org/ 10.1016/j.paid.2008.12.010

Denson, T. F., Grisham, J. R., \& Moulds, M. L. (2011). Cognitive reappraisal increases heart rate variability in response to an anger provocation. Motivation and Emotion, 35(1), 14-22. http://dx.doi.org/ 10.1007/s11031-011-9201-5

English, T., \& John, O. P. (2013). Understanding the social effects of emotion regulation: The mediating role of authenticity for individual differences in suppression. Emotion, 13(2), 314-329. https://doi.org/10.1037/a0029847

Fahy, K. M., Lee, A. J., Milne, B. (2017) New Zealand socio-economic index 2013. Statistics New Zealand.

Franchow, E. I., \& Suchy, Y. (2015). Naturally-occurring emotional suppression in daily life depletes executive functioning. Emotion, 15(1), 78-89. https://doi.org/10.1037/emo0000013

Fraser, G. (2018). Statistical standard for coding gender identity (Revised). NZAVS Technical Documents, e27.

Fredrickson, B. L., Tugade, M. M., Waugh, C. E., \& Larkin, G. R. (2003). What good are positive emotions in crises? A prospective study of resilience and emotions following the terrorist attacks on the United States on September 11th, 2001. Journal of personality and social psychology, 84(2), 365-376. https://doi.org/10.1037//00223514.84 .2 .365

Fritz, H. L. (1999). Rumination and adjustment to a first coronary event. Psychosomatic Medicine, 61(1), 105. 
Ford, B. Q., Gross, J. J., \& Gruber, J. (2019). Broadening our field of view: The role of emotion polyregulation. Emotion Review, 11(3), 197-208. https://doi.org/10.1177/1754073919850314

Ford, B. Q., Karnilowicz, H. R., \& Mauss, I. B. (2017). Understanding reappraisal as a multicomponent process: The psychological health benefits of attempting to use reappraisal depend on reappraisal success. Emotion, 17(6), 905911. https://doi.org/10.1037/emo0000310

Glass, K., Flory, K., Hankin, B. L., Kloos, B., \& Turecki, G. (2009). Are coping strategies, social support, and hope associated with psychological distress among Hurricane Katrina survivors? Journal of Social and Clinical Psychology, 28(6), 779-795. https://doi.org/10.1521/jscp.2009.28.6.779

Goldberg, L. S., \& Grandey, A. A. (2007). Display rules versus display autonomy: Emotion regulation, emotional exhaustion, and task performance in a call center simulation. Journal of Occupational Health Psychology, 12, 301-318. http://dx.doi.org/10.1037/1076-8998.12.3.301

Gratz, K. L., \& Roemer, L. (2004). Multidimensional assessment of emotion regulation and dysregulation: Development, factor structure, and initial validation of the difficulties in emotion regulation scale. Journal of Psychopathology and Behavioral Assessment, 26, 41-54. http://dx.doi.org/10.1023/B:JOBA.0000007455.08539.94

Graves, P. L., Mead, L. A., Wang, N. Y., Liang, K. Y., \& Klag, M. J. (1994). Temperament as a potential predictor of mortality: evidence from a 41-year prospective study. Journal of behavioral medicine, 17(2), 111-126.

Grimm, A., L. Hulse, M. Preiss, and S. Schmidt (2012). Post- and peri-traumatic stress in disaster survivors: an explorative study about the influence of individual and event characteristics across different types of disasters. European Journal of Psychotraumatology, 3(7382), 1-9. http://dx.doi.org/ 10.3402/ejpt.v3i0.7382 
Gross, J. J. (1998). The emerging field of emotion regulation: An integrative review. Review of general psychology, 2(3), 271-299. http://dx.doi.org/ 10.1037/1089-2680.2.3.271

Gross, J. J. (2013). Emotion regulation: taking stock and moving forward. Emotion, 13(3), 359-365. https://doi.org/10.1037/a0032135

Gross, J. J. (2015). Emotion regulation: Current status and future prospects. Psychological inquiry, 26(1), 1-26. http://dx.doi.org/ 10.1080/1047840X.2014.940781

Gross, J. J., \& John, O. P. (2003). Individual differences in two emotion regulation processes: implications for affect, relationships, and well-being. Journal of Personality and Social Psychology, 85(2), 348-362. https://doi.org/10.1037/0022-3514.85.2.348.

Gross, J. J., \& Levenson, R. W. (1993). Emotional suppression: Physiology, self-report, and expressive behavior. Journal of Personality and Social Psychology, 64, 970-986. http://dx.doi.org/10.1037/0022-3514 .64.6.970

Gross, J. J., \& Levenson, R. W. (1997). Hiding feelings: the acute effects of inhibiting negative and positive emotion. Journal of Abnormal Psychology, 106(1), 95103. https://doi.org/10.1037/0021-843X.106.1.95

Gross, J. J., \& Muñoz, R. F. (1995). Emotion regulation and mental health. Clinical psychology: Science and practice, 2(2), 151-164. http://dx.doi.org/ 10.1111/j.14682850.1995.tb00036.x

Gruber, J., Prinstein, M. J., Clark, L. A., Rottenberg, J., Abramowitz, J. S., Albano, A. M., Aldao, A., Borelli, J. L., Chung, T., Davila, J., Forbes, E. E., Gee, D. G., Hall, G. C. N., Hallion, L. S., Hinshaw, S. P., Hofmann, S. G., Hollon, S. D., Joormann, J., Kazdin, A. E., Klein, D. N., La Greca, A. M., Levenson, R. W., MacDonald, A. W., III, McKay, D., McLaughlin, K. A., Mendle, J., Miller, A. B., Neblett, E. W., Nock, M., Olatunji, B. O., Persons, J. B., Rozek, D. C., Schleider, J. L., Slavich, G. M., Teachman, B. A., Vine, V., \& Weinstock, L. M. (2020). Mental Health and Clinical Psychological Science in the Time of COVID-19: Challenges, Opportunities, and a 
Call to Action. American Psychologist. Advance online publication. http://dx.doi.org/10.1037/amp0000707

Holmes, E. A., O’Connor, R. C., Perry, V. H., Tracey, I., Wessely, S., Arseneault, L. ,... Bullmore, E. (2020). Multidisciplinary research priorities for the COVID-19 pandemic: A call for action for mental health science. The Lancet Psychiatry, 7, 547560. http://dx.doi.org/10.1016/S2215-0366(20)30168-1

Hopp, H., Troy, A. S., \& Mauss, I. B. (2011). The unconscious pursuit of emotion regulation: Implications for psychological health. Cognition and Emotion, 25(3), 532-545. http://dx.doi.org/ 10.1080/02699931.2010.532606

Impett, E. A., Kogan, A., English, T., John, O., Oveis, C., Gordon, A. M., \& Keltner, D. (2012). Suppression sours sacrifice: Emotional and relational costs of suppressing emotions in romantic relationships. Personality and Social Psychology Bulletin, 38(6), 707-720. http://dx.doi.org/ 10.1177/0146167212437249

Jacobson, N. S., \& Truax, P. (1991). Clinical significance: A statistical approach to defining meaningful change in psychotherapy research. In A. E. Kazdin (Ed.), Methodological issues \& strategies in clinical research, 631-648. American Psychological Association. https://doi.org/10.1037/10109-042

Kamo, Y., Henderson, T. L., \& Roberto, K. A. (2011). Displaced older adults' reactions to and coping with the aftermath of Hurricane Katrina. Journal of family issues, 32(10), 1346-1370. http://dx.doi.org/ 10.1177/0192513X11412495

Kashdan, T. B., Elhai, J. D., \& Breen, W. E. (2008). Social anxiety and disinhibition: an analysis of curiosity and social rank appraisals, approach-avoidance conflicts, and disruptive risk-taking behavior. Journal of anxiety disorders, 22(6), 925-939. https://doi.org/10.1016/j.janxdis.2007.09.009

Kelly, A. E., \& Kahn, J. H. (1994). Effects of suppression of personal intrusive thoughts. Journal of Personality and Social Psychology, 66(6), 998-1006. 
Kenny, D. A., Kashy, D. A., \& Cook, W. L. (2006). Dyadic data analysis. New York, NY: Guilford Press.

Kishore, V., Theall, K. P., Robinson, W., Pichon, J., Scribner, R., Roberson, E., \& Johnson, S. (2008). Resource loss, coping, alcohol use, and posttraumatic stress symptoms among survivors of Hurricane Katrina: a cross-sectional study. American journal of disaster medicine, 3(6), 345-357.

Kivity, Y., Tamir, M., \& Huppert, J. D. (2016). Self-acceptance of negative emotions: The positive relationship with effective cognitive reappraisal. International Journal of Cognitive Therapy, 9, 1-16. http://dx.doi.org/10.1521/ijct_2016_09_10

Kubzansky, L. D., Park, N., Peterson, C., Vokonas, P., \& Sparrow, D. (2011). Healthy psychological functioning and incident coronary heart disease: the importance of selfregulation. Archives of General psychiatry, 68(4), 400-408.

http://dx.doi.org/10.1001/archgenpsychiatry.2011.23

Low, R. S., Overall, N. C., Cross, E. J., \& Henderson, A. M. (2019). Emotion regulation, conflict resolution, and spillover on subsequent family functioning. Emotion, 19(7), 1162-1182. https://doi.org/10.1037/emo0000519

Low, R. S., Overall, N. C., Hammond, M. D., \& Girme, Y. U. (2017). Emotional suppression during personal goal pursuit impedes goal strivings and achievement. Emotion, 17(2), 208-223. https://doi.org/10.1037/emo0000218

Lyubomirsky, S., \& Nolen-Hoeksema, S. (1995). Effects of self-focused rumination on negative thinking and interpersonal problem solving. Journal of Personality and Social Psychology, 69, 176-190. http://dx .doi.org/10.1037/0022-3514.69.1.176

Marroquín, B., Tennen, H., \& Stanton, A. L. (2017). Coping, emotion regulation, and wellbeing: Intrapersonal and interpersonal processes. In The happy mind: Cognitive contributions to well-being (pp. 253-274). Springer, Cham. 
Mauss, I. B., Cook, C. L., Cheng, J. Y., \& Gross, J. J. (2007). Individual differences in cognitive reappraisal: Experiential and physiological responses to an anger provocation. International Journal of Psychophysiology, 66(2), 116-124. https://doi.org/10.1016/j.ijpsycho.2007.03.017

Mauss, I. B., Levenson, R. W., McCarter, L., Wilhelm, F. H., \& Gross, J. J. (2005). The tie that binds? Coherence among emotion experience, behavior, and physiology. Emotion, 5(2), 175-190. https://doi.org/10.1037/1528-3542.5.2.175

Menard, S. (1995). Applied logistic regression analysis. Sage University Paper Series on Quantitative Applications in the Social Sciences, 7-106. Thousand Oaks, CA: Sage. McKibbin, W., \& Fernando, R. (2020). The global macroeconomic impacts of COVID-19: Seven scenarios. Centre for Applied Macroeconomic Analysis; The Australian National University. https://cama.crawford.anu.edu.au/publication/camaworkingpaper-series/16221/global-macroeconomic-impacts-covid-19-seven-scenarios McNally, R. J. (1995). Automaticity and the anxiety disorders. Behaviour Research and Therapy, 33(7), 747-754. Scopus. https://doi.org/10.1016/0005-7967(95)00015-P

Mellings, T. M., \& Alden, L. E. (2000). Cognitive processes in social anxiety: The effects of self-focus, rumination and anticipatory processing. Behaviour research and therapy, 38(3), 243-257. https://doi.org/10.1016/S0005-7967(99)00040-6

Mesidor, J. K., \& Sly, K. F. (2016). Factors that contribute to the adjustment of international students. Journal of International Students, 6(1), 262-282.

Moulds, M. L., Kandris, E., Starr, S., \& Wong, A. C. (2007). The relationship between rumination, avoidance and depression in a non-clinical sample. Behaviour research and therapy, 45(2), 251-261. https://doi.org/10.1016/j.brat.2006.03.003

Muthén, L.K. and Muthén, B.O. (1998-2017). Mplus User's Guide. Eighth Edition. Los Angeles, CA: Muthén \& Muthén 
Myers, R. (1990). Classical and modern regression with applications (2nd ed.). Boston: Duxbury.

Naragon-Gainey, K., McMahon, T. P., \& Chacko, T. P. (2017). The structure of common emotion regulation strategies: A meta-analytic examination. Psychological Bulletin, 143(4), 384-427. https://doi.org/10.1037/bu10000093

Neff, L. A., \& Broady, E. F. (2011). Stress resilience in early marriage: Can practice make perfect? Journal of Personality and Social Psychology, 101(5), 10501067. https://doi.org/10.1037/a0023809

Nolen-Hoeksema, S. (1987). Sex differences in unipolar depression: Evidence and theory. Psychological Bulletin, 101, 259-282. http://dx.doi .org/10.1037/0033-2909.101.2.259

Nolen-Hoeksema, S. (1991). Responses to depression and their effects on the duration of depressive episodes. Journal of abnormal psychology, 100(4), 569-582.

Nolen-Hoeksema, S. (1998). The other end of the continuum: The costs of rumination. Psychological Inquiry, 9(3), 216-219.

Nolen-Hoeksema, S. (2000). The role of rumination in depressive disorders and mixed anxiety/depressive symptoms. Journal of Abnormal Psychology, 109(3), 504511. https://doi.org/10.1037/0021-843X.109.3.504

Nolen-Hoeksema, S. (2004). The response styles theory. Depressive rumination: Nature, theory and treatment, 107-123.

Olatunji, B. O., Naragon-Gainey, K., \& Wolitzky-Taylor, K. B. (2013). Specificity of rumination in anxiety and depression: A multimodal meta-analysis. Clinical Psychology: Science and Practice, 20(3), 225-257. https://doi.org/10.1037/h0101719

Perneger, T. V. (1998). What's wrong with Bonferroni adjustments. Bmj, 316(7139), 12361238. https://doi.org/10.1136/bmj.316.7139.1236 
Peters, B. J., \& Jamieson, J. P. (2016). The consequences of suppressing affective displays in romantic relationships: A challenge and threat perspective. Emotion, 16(7), 10501066. https://doi.org/10.1037/emo0000202

Peters, B. J., Overall, N. C., Cameron, L. D., Hammond, M. D., Low, R. S. T., \& Girme, Y. U. (2020). Do habitual emotional suppression measures predict response-focused situational suppression during social interactions? Emotion, 20(6), 10051019. https://doi.org/10.1037/emo0000620

Peters, B. J., Overall, N. C., \& Jamieson, J. P. (2014). Physiological and cognitive consequences of suppressing and expressing emotion in dyadic interactions. International Journal of Psychophysiology, 94, 100-107. http://dx.doi.org/10.1016/j.ijpsycho.2014.07.015

Quoidbach, J., Berry, E. V., Hansenne, M., \& Mikolajczak, M. (2010). Positive emotion regulation and well-being: Comparing the impact of eight savoring and dampening strategies. Personality and individual differences, 49(5), 368-373. https://doi.org/10.1016/j.paid.2010.03.048

Radloff, L. S. (1977). The CES-D scale: A self-report depression scale for research in the general population. Applied psychological measurement, 1(3), 385-401. https://doi.org/10.1177/014662167700100306

Radstaak, M., Geurts, S. A., Brosschot, J. F., Cillessen, A. H., \& Kompier, M. A. (2011). The role of affect and rumination in cardiovascular recovery from stress. International Journal of Psychophysiology, 81(3), 237-244. https://doi.org/10.1016/j.ijpsycho.2011.06.017

RAND (n.d.). Retrieved from https://www.rand.org/health-care/surveys_tools/mos/36-itemshort-form/scoring.html 
Rettie, H., \& Daniels, J. (2020). Coping and tolerance of uncertainty: Predictors and mediators of mental health during the COVID-19 pandemic. American Psychologist. Advance online publication. http://dx.doi.org/10.1037/amp0000710

Richards, J. M., \& Gross, J. J. (1999). Composure at any cost? The cognitive consequences of emotion suppression. Personality and Social Psychology Bulletin, 25, 1033-1044. http://dx.doi.org/10 .1177/01461672992511010

Richards, J. M., \& Gross, J. J. (2000). Emotion regulation and memory: The cognitive costs of keeping one's cool. Journal of Personality and Social Psychology, 79, 410-424. http://dx.doi.org/10.1037/0022-3514 .79.3.410

Rosenthal, R., \& Rosnow, R. L. (2008). Essentials of behavioral research: Methods and data analysis (3rd ed.). McGraw-Hill.

Rucci, P., Gherardi, S., Tansella, M., Piccinelli, M., Berardi, D., Bisoffi, G., Corsino, M. A., \& Pini, S. (2003). Subthreshold psychiatric disorders in primary care: prevalence and associated characteristics. Journal of affective disorders, 76(1-3), 171-181. https://doi.org/10.1016/s0165-0327(02)00087-3

Sibley, C. G., Greaves, L. M., Satherley, N., Wilson, M. S., Overall, N. C., Lee, C. H. J., . . . Barlow, F. K. (2020). Effects of the COVID-19 pandemic and nationwide lockdown on trust, attitudes toward government, and well-being. American Psychologist, 75(5), 618-630. http://dx.doi.org/10.1037/amp0000662

Smith, A. M., Willroth, E. C., Gatchpazian, A., Shallcross, A. J., Feinberg, M., \& Ford, B. Q. (2021). Coping with health threats: The costs and benefits of managing emotions. Psychological Science, 32(7), 1011-1023. https://doi.org/10.1177/09567976211024260

Spasojevic, J., Alloy, L. B., Abramson, L. Y., Maccoon, D., \& Robinson, M. S. (2004). Reactive rumination: Outcomes, mechanisms, and developmental antecedents. Depressive rumination: Nature, theory and treatment, 43-58. 
Srivastava, S., Tamir, M., McGonigal, K. M., John, O. P., \& Gross, J. J. (2009). The social costs of emotional suppression: A prospective study of the transition to college. Journal of Personality and Social Psychology, 96, 883-897. http://dx.doi.org/10.1037/a0014755

Suls, J., \& Bunde, J. (2005). Anger, anxiety, and depression as risk factors for cardiovascular disease: the problems and implications of overlapping affective dispositions. Psychological bulletin, 131(2), 260-300

Takano, K., Sakamoto, S., \& Tanno, Y. (2011). Ruminative and reflective forms of selffocus: Their relationships with interpersonal skills and emotional reactivity under interpersonal stress. Personality and Individual Differences, 51(4), 515-520. https://doi.org/10.1016/j.paid.2011.05.010

Tamagawa, R., Giese-Davis, J., Speca, M., Doll, R., Stephen, J., \& Carlson, L. E. (2013). Trait mindfulness, repression, suppression, and self-reported mood and stress symptoms among women with breast cancer. Journal of clinical psychology, 69(3), 264-277. https://doi.org/ 10.1002/jclp.21939

Thomsen, D. K., Mehlsen, M. Y., Olesen, F., Hokland, M., Viidik, A., Avlund, K., \& Zachariae, R. (2004). Is there an association between rumination and self-reported physical health? A one-year follow-up in a young and an elderly sample. Journal of Behavioral Medicine, 27(3), 215-231. https://doi.org/10.1023/B:JOBM.0000028496.41492.34

Thomson, R. A., Overall, N. C., Cameron, L. D., \& Low, R. S. (2018). Perceived regard, emotional suppression during conflict, and conflict resolution. Journal of Family Psychology, 32(6), 722-732. https://doi.org/10.1037/fam0000429

Troy, A. S., Shallcross, A. J., \& Mauss, I. B. (2013). A person-by-situation approach to emotion regulation: Cognitive reappraisal can either help or hurt, depending on the 
context. Psychological science, 24(12), 2505-2514.

https://doi.org/10.1177/0956797613496434

Wang, K., Wei, D., Yang, J., Xie, P., Hao, X., \& Qiu, J. (2015). Individual differences in rumination in healthy and depressive samples: association with brain structure, functional connectivity and depression. Psychological medicine, 45(14), 2999-3008.

Ware, J. E., Jr., \& Sherbourne, C.D. (1992). The MOS 36-Item Short-Form Health Survey (SF-36): I. Conceptual Framework and Item Selection. Medical Care, 30:473-483.

Watkins, E. D., \& Baracaia, S. (2002). Rumination and social problem-solving in depression. Behaviour research and therapy, 40(10), 1179-1189. https://doi.org/ 10.1016/S0005-7967(01)00098-5

Watkins, E., \& Brown, R. G. (2002). Rumination and executive function in depression: An experimental study. Journal of Neurology, Neurosurgery, and Psychiatry, 72, 400402. http://dx.doi.org/10.1136/jnnp.72.3.400

Webb, T. L., Miles, E., \& Sheeran, P. (2012). Dealing with feeling: a meta-analysis of the effectiveness of strategies derived from the process model of emotion regulation. Psychological Bulletin, 138(4), 775808. https://doi.org/10.1037/a0027600

Wenzlaff, R. M., \& Luxton, D. D. (2003). The role of thought suppression in depressive rumination. Cognitive Therapy and Research, 27, 293-308. http://dx.doi.org/10.1023/A:1023966400540

Yook, K., Kim, K. H., Suh, S. Y., \& Lee, K. S. (2010). Intolerance of uncertainty, worry, and rumination in major depressive disorder and generalized anxiety disorder. Journal of anxiety disorders, 24(6), 623-628. https://doi.org/10.1016/j.janxdis.2010.04.003

Zacher, H., \& Rudolph, C. W. (2020). Individual differences and changes in subjective wellbeing during the early stages of the COVID-19 pandemic. American Psychologist. Advance online publication. http://dx.doi.org/10.1037/amp0000702 
Zaki, J., \& Williams, W. C. (2013). Interpersonal emotion regulation. Emotion, 13(5), 803810. https://doi.org/10.1037/a0033839

Zoccola, P. M., Dickerson, S. S., \& Zaldivar, F. P. (2008). Rumination and cortisol responses to laboratory stressors. Psychosomatic medicine, 70(6), 661-667. http://dx.doi.org/ 10.1097/PSY.0b013e31817bbc77 
Table 1.

Descriptive Statistics across all primary measures in Study 1

\begin{tabular}{lcccccc}
\hline & \multicolumn{3}{c}{ Pre-Lockdown } & \multicolumn{3}{c}{ Lockdown } \\
Measures & Range & $M$ & $S D$ & Range & $M$ & $S D$ \\
\hline Depressive symptoms & $0-28$ & 6.34 & 4.52 & $0-24$ & 7.62 & 5.10 \\
Emotional well-being & $10-100$ & 74.54 & 15.20 & $25-100$ & 74.04 & 16.46 \\
Energy (Low Fatigue) & $0-100$ & 53.32 & 18.37 & $0-100$ & 54.18 & 20.49 \\
General health & $5-100$ & 66.85 & 18.91 & $10-100$ & 69.62 & 17.73 \\
Rumination & - & - & - & $1-7$ & 2.28 & 1.30 \\
Emotional suppression & - & - & - & $1-7$ & 3.08 & 1.45 \\
Cognitive reappraisal & - & - & - & $1-7$ & 4.23 & 1.64 \\
Emotion control difficulties & - & - & - & $1-6.75$ & 2.42 & 1.32 \\
Stress & - & - & - & $1-5.58$ & 2.66 & 0.96 \\
Perceived support & - & - & - & $1-5$ & 3.28 & 0.90 \\
\hline Note. Depressin
\end{tabular}

Note. Depressive symptoms are summed index that can range from 0-30. All other psychological and physical health assessments are scored on scales of 1-100. All other lockdown measures are assessed on 1-7 scales. Pre-lockdown and lockdown assessments of emotional well-being, energy/fatigue and general health are not directly comparable because the pre-lockdown assessments asked participants to reflect on their health across the prior four weeks, whereas the lockdown assessments were specific to the lockdown period. 
Table 2.

Correlations Across All Measures in Study 1

\begin{tabular}{|c|c|c|c|c|c|c|c|c|c|}
\hline Measures & 1. & 2. & 3. & 4. & 5. & 6. & 7. & 8. & 9. \\
\hline 1. Depressive symptoms & $.28^{* *}$ & $-.79^{* *}$ & $-.67^{* *}$ & $-.44^{* *}$ & & & & & \\
\hline 2. Emotional well-being & $-.82^{* *}$ & $.34^{* *}$ & $.66^{* *}$ & $.37^{* *}$ & & & & & \\
\hline 3. Energy (Low Fatigue) & $-.69^{* *}$ & $.73^{* *}$ & $.47^{* *}$ & $.51^{* *}$ & & & & & \\
\hline 4. General health & $-.30^{* *}$ & $.33^{* *}$ & $.37^{* *}$ & $.64^{* *}$ & & & & & \\
\hline 5. Rumination & $.75^{* *}$ & $-.74^{* *}$ & $-.64^{* *}$ & $-.26^{* *}$ & & & & & \\
\hline 6. Emotional suppression & $.60^{* *}$ & $-.56^{* *}$ & $-.48^{* *}$ & $-.21^{* *}$ & $.63^{* *}$ & & & & \\
\hline 8. Emotion control difficulties & $.72^{* *}$ & $-.71^{* *}$ & $-.62^{* *}$ & $-.22^{* *}$ & $.81^{* *}$ & $.58^{* *}$ & $.33^{* *}$ & & \\
\hline 9. Stress & $.65^{* *}$ & $-.62^{* *}$ & $-.58^{* *}$ & $-.33^{* *}$ & $.64^{* *}$ & $.55^{* *}$ & $.39^{* *}$ & $.61^{* *}$ & \\
\hline 10. Perceived support & $-.21^{* *}$ & $.21^{* *}$ & $.18^{* *}$ & $.13^{*}$ & $-.17^{* *}$ & $-.21^{* *}$ & -.06 & $-.16^{* *}$ & $-.22^{* *}$ \\
\hline
\end{tabular}

Note. Correlations for pre-lockdown measures are above the diagonal. Correlations across measures assessed during the lockdown are below the diagonal. Bold correlations on the diagonal represent the within-measure associations across pre-lockdown and lockdown assessments. ${ }^{*} p<.05 .{ }^{* *} p<.01$. 
Table 3.

The Associations between Emotion Regulation Strategies and Psychological and Physical Health during Lockdown in Study 1

\begin{tabular}{|c|c|c|c|c|c|c|c|c|c|c|c|c|c|c|c|c|c|c|c|c|}
\hline \multirow[t]{2}{*}{ Predictors } & \multicolumn{5}{|c|}{ Depressive Symptoms } & \multicolumn{5}{|c|}{ Emotional Well-being } & \multicolumn{5}{|c|}{ Energy (Low Fatigue) } & \multicolumn{5}{|c|}{ General Health } \\
\hline & $B$ & $95 \% C I$ & $t$ & $p$ & $r$ & $B$ & $95 \% C I$ & $t$ & $p$ & $r$ & $B$ & $95 \% C I$ & $t$ & $p$ & $r$ & $B$ & $95 \% C I$ & $t$ & $p$ & $r$ \\
\hline \multicolumn{21}{|c|}{ Three Separate Models Assessing the Effects of Each Emotion Regulation Strategy } \\
\hline Rumination & 2.74 & $2.483,3.006$ & 20.64 & $<.001$ & .74 & -8.75 & $-9.637,-7.864$ & -19.41 & $<.001$ & .72 & -8.68 & $-9.845,-7.514$ & -14.64 & $<.001$ & .61 & -1.64 & $-2.716,-.562$ & -2.99 & .003 & .16 \\
\hline $\begin{array}{l}\text { Emotional } \\
\text { suppression }\end{array}$ & 1.91 & $1.613,2.200$ & 12.76 & $<.001$ & .56 & -5.73 & $-6.696,-4.766$ & -11.68 & $<.001$ & .52 & -5.81 & $-6.953,-4.658$ & -9.95 & $<.001$ & .47 & -1.20 & $-2.178,-.228$ & -2.43 & .016 & .13 \\
\hline $\begin{array}{l}\text { Cognitive } \\
\text { reappraisal }\end{array}$ & .98 & $.697,1.258$ & 6.87 & $<.001$ & .35 & -2.75 & $-3.671,-1.826$ & -5.86 & $<.001$ & .30 & -3.80 & $-4.850,-2.752$ & -7.13 & $<.001$ & .36 & -.86 & $-1.720,-.005$ & -1.98 & .049 & .10 \\
\hline \multicolumn{21}{|c|}{ One Model Assessing the Unique Effects of Each Emotion Regulation Strategy } \\
\hline Rumination & 2.37 & $2.047,2.697$ & 14.35 & $<.001$ & .62 & -7.72 & $-8.822,-6.615$ & -13.76 & $<.001$ & .60 & -7.33 & $-8.803,-5.867$ & -9.83 & $<.001$ & .46 & -1.28 & $-2.667, .100$ & -1.82 & .069 & .10 \\
\hline $\begin{array}{l}\text { Emotional } \\
\text { suppression }\end{array}$ & .55 & $.228, .871$ & 3.36 & .001 & .18 & -1.77 & $-2.852,-.692$ & -3.23 & .001 & .17 & -1.13 & $-2.549, .296$ & -1.56 & .120 & .08 & -.26 & $-1.625,1.101$ & -0.38 & .705 & .02 \\
\hline $\begin{array}{l}\text { Cognitive } \\
\text { reappraisal }\end{array}$ & .03 & $-.210, .263$ & 0.22 & .827 & .01 & .32 & $-.480,1.117$ & 0.78 & .433 & .04 & -1.08 & $-2.134,-.028$ & -2.02 & .044 & .11 & -.37 & $-1.373, .635$ & -0.72 & .471 & .04 \\
\hline
\end{tabular}

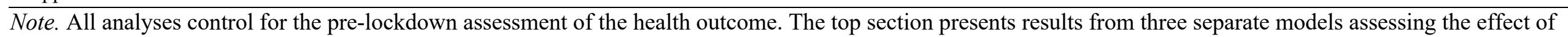

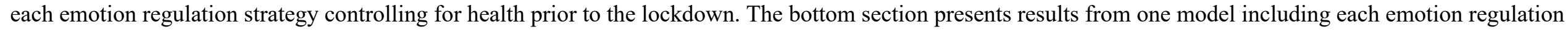

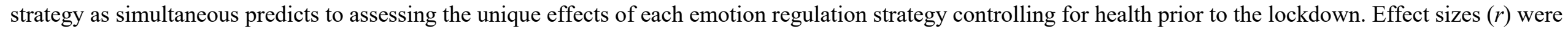

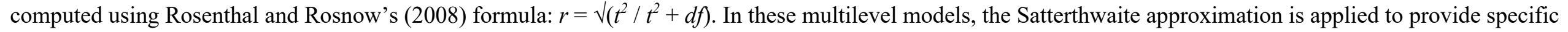

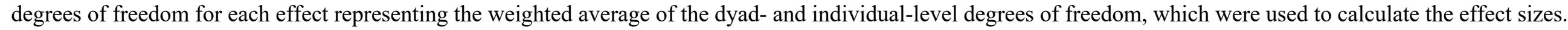


Table 4.

Controlling for Alternative Explanations for the Links Between Emotion Regulation Strategies and Psychological and Physical Health during Lockdown in Study 1

\begin{tabular}{|c|c|c|c|c|c|c|c|c|c|c|c|c|c|c|c|}
\hline \multirow[t]{2}{*}{ Predictors } & \multicolumn{5}{|c|}{ Depressive Symptoms } & \multicolumn{5}{|c|}{ Emotional Well-being } & \multicolumn{5}{|c|}{ Energy (Low Fatigue) } \\
\hline & $B$ & $95 \% C I$ & $t$ & $p$ & $r$ & $B$ & $95 \% C I$ & $t$ & $p$ & $r$ & $B$ & $95 \% C I$ & $t$ & $p$ & $r$ \\
\hline \multicolumn{16}{|c|}{ Controlling for Emotion Control Difficulties } \\
\hline Rumination & 1.60 & $1.159,2.040$ & 7.14 & $<.001$ & .36 & -5.13 & $-6.623,-3.644$ & -6.78 & $<.001$ & .34 & -4.49 & $-6.462,-2.520$ & -4.48 & $<.001$ & .23 \\
\hline Emotional suppression & .44 & $.122, .756$ & 2.73 & .007 & .15 & -1.36 & $-2.424,-.304$ & -2.53 & .012 & .13 & -.68 & $-2.089, .729$ & -0.95 & .343 & .05 \\
\hline Cognitive reappraisal & .04 & $-.186, .274$ & 0.38 & .706 & .02 & .26 & $-.516,1.034$ & 0.66 & .511 & .03 & -1.13 & $-2.160,-.096$ & -2.15 & .032 & .11 \\
\hline $\begin{array}{l}\text { Emotion Control } \\
\text { Difficulties }\end{array}$ & 1.07 & $.644,1.494$ & 4.95 & $<.001$ & .26 & -3.54 & $-4.955,-2.119$ & -4.91 & $<.001$ & .25 & -4.00 & $-5.897,-2.104$ & -4.15 & $<.001$ & .21 \\
\hline \multicolumn{16}{|l|}{ Controlling for Stress } \\
\hline Rumination & 1.91 & $1.562,2.258$ & 10.81 & $<.001$ & .51 & -6.24 & $-7.419,-5.063$ & -10.42 & $<.001$ & .49 & -5.47 & $-7.051,-3.896$ & -6.82 & $<.001$ & .34 \\
\hline Emotional suppression & .40 & $.083, .711$ & 2.49 & .013 & .13 & -1.23 & $-2.278,-.174$ & -2.29 & .022 & .12 & -.49 & $-1.891, .911$ & -0.69 & .492 & .04 \\
\hline Cognitive reappraisal & -.05 & $-.282, .177$ & -0.45 & .653 & .02 & .58 & $-.192,1.351$ & 1.48 & .140 & .08 & -.72 & $-1.753, .303$ & -1.39 & .166 & .07 \\
\hline Stress & 1.32 & $.863,1.770$ & 5.71 & $<.001$ & .29 & -4.28 & $-5.793,-2.770$ & -5.57 & $<.001$ & .28 & -5.30 & $-7.305,-3.302$ & -5.21 & $<.001$ & .27 \\
\hline \multicolumn{16}{|c|}{ Controlling for Perceived Support } \\
\hline Rumination & 2.36 & $2.033,2.684$ & 14.26 & $<.001$ & .61 & -7.69 & $-8.798,-6.592$ & -13.72 & $<.001$ & .60 & -7.32 & $-8.791,-5.850$ & -9.79 & $<.001$ & .46 \\
\hline Emotional suppression & .52 & $.197, .844$ & 3.16 & .002 & .17 & -1.67 & $-2.758,-.583$ & -3.02 & .003 & .16 & -1.07 & $-2.507, .364$ & -1.47 & .143 & .08 \\
\hline Cognitive reappraisal & .04 & $-.200, .272$ & 0.30 & .765 & .02 & .279 & $-.521,1.078$ & 0.69 & .493 & .04 & -1.09 & $-2.150, .040$ & -2.04 & .042 & .11 \\
\hline Perceived Support & -.31 & $-.706, .090$ & -1.52 & .129 & .08 & .906 & $-.384,2.197$ & 1.38 & .168 & .08 & .50 & $-1.249,2.241$ & 0.56 & .576 & .03 \\
\hline
\end{tabular}

Note. Analyses controlling for alternative explanations were only conducted when the emotion regulation strategy had illustrated unique associations in models

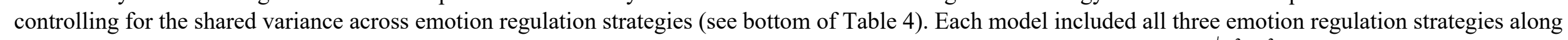
with the alternative explanation variable. Effect sizes $(r)$ were computed using Rosenthal and Rosnow's (2008) formula: $r=\sqrt{ }\left(t^{2} / t^{2}+d f\right)$. In these multilevel models, the Satterthwaite approximation is applied to provide specific degrees of freedom for each effect representing the weighted average of the dyad- and individual-level degrees of freedom, which were used to calculate the effect sizes. 
Table 5.

Demographic Information for Study $2(N=1607)$ Included as Covariates across Analyses

\begin{tabular}{lcc}
\hline Variable & $\%(n)$ & $M(S D)$ \\
\hline Ethnic Identification (compared to NZ & \\
European/Pākehā majority) & & \\
$\quad$ Māori & $9.5 \%(153)$ & \\
$\quad$ Pacific & $2.0 \%(32)$ & \\
$\quad$ Asian & $3.4 \%(54)$ & \\
Gender & \multicolumn{3}{c}{$65.2 \%(1048)$} & \\
$\quad$ Women & $34.8 \%(559)$ & \\
$\quad$ Men & & $51.57(13.40)$ \\
Age & & $5.54(2.70)$ \\
Education (0 low - 10 high) & $79.7 \%(1280)$ & \\
Employed & & \\
New Zealand Deprivation Index (1 low - 10 high) & \\
New Zealand Socioeconomic Index (10 low - 90 high) & & \\
Live in Major Urban Area & $82.7 \%(13)$ \\
In a serious relationship & $73.9 \%(1187)$ & \\
Parent & $71.6 \%(1151)$ & \\
Born in New Zealand & $79.6 \%(1279)$ & \\
\hline
\end{tabular}


Table 6.

Descriptive Statistics and Mean Differences Between the Pre-Lockdown and Lockdown Samples Across Measures in Study 2

\begin{tabular}{lcccrrr}
\hline Measures & \multicolumn{7}{c}{$\begin{array}{c}\text { Pre- } \\
\text { lockdown }\end{array}$} & Lockdown & & \\
Range & $M(S D)$ & $M(S D)$ & \multicolumn{1}{c}{$t$} & \multicolumn{1}{c}{$d f$} & \multicolumn{1}{c}{$p$} \\
\hline Psychological Distress & $0-4$ & $0.88(0.65)$ & $0.91(0.63)$ & -1.65 & 1594 & .099 \\
Personal well-being & $0-10$ & $7.09(1.65)$ & $7.07(1.68)$ & 0.68 & 1606 & .494 \\
Fatigue (Low Energy) & $0-4$ & $1.65(1.07)$ & $1.48(1.04)$ & 7.63 & 1590 & $<.001$ \\
General health & $1-7$ & $5.03(1.14)$ & $5.04(1.18)$ & -1.23 & 1605 & .221 \\
Rumination & $0-4$ & $0.82(0.97)$ & $0.80(0.94)$ & 1.02 & 1623 & .308 \\
Emotional suppression & $1-7$ & $4.21(1.64)$ & $4.23(1.62)$ & -0.34 & 1634 & .732 \\
Cognitive reappraisal & $1-7$ & $4.80(1.41)$ & $4.93(1.39)$ & -3.27 & 1539 & .001 \\
Emotion control difficulties & $1-7$ & $2.78(1.59)$ & $2.82(1.63)$ & -0.84 & 1618 & .401 \\
Perceived support & $1-7$ & $5.96(1.11)$ & $5.99(1.15)$ & -1.24 & 1646 & .216 \\
\hline
\end{tabular}

Note. Ns and df varied due to small amount of missing data on some variables. 
Table 7.

Correlations Across All Measures in Study 2

\begin{tabular}{|c|c|c|c|c|c|c|c|c|c|}
\hline Measures & 1. & 2. & 3. & 4. & 5. & 6. & 7. & 8. & 9. \\
\hline 1. Depressive symptoms & $.66^{* *}$ & $-.50^{* *}$ & $.57^{* *}$ & $-.35^{* *}$ & $.70^{* *}$ & $.26^{* *}$ & $-.19^{* *}$ & $.50^{* *}$ & $-.41^{* *}$ \\
\hline 2. Personal well-being & $-.44^{* *}$ & $.71^{* *}$ & $-.37^{* *}$ & $.53^{* *}$ & $-.37^{* *}$ & $-.21^{* *}$ & $.16^{* *}$ & $-.30^{* *}$ & $.51^{* *}$ \\
\hline 3. Energy/Fatigue & $.56^{* *}$ & $-.33^{* *}$ & $.59^{* *}$ & $-.31^{* *}$ & $.39^{* *}$ & $.16^{* *}$ & $-.10^{* *}$ & $.27^{* *}$ & $-.24^{* *}$ \\
\hline 4. General health & $-.35^{* *}$ & $.49^{* *}$ & $-.33^{* *}$ & $.70^{* *}$ & $-.24^{* *}$ & $-.15^{* *}$ & $.13^{* *}$ & $-.25^{* *}$ & $.27^{* *}$ \\
\hline 5. Rumination & $.66^{* *}$ & $-.31^{* *}$ & $.37^{* *}$ & $-.23^{* *}$ & $.56^{* *}$ & $.20^{* *}$ & $-.14^{* *}$ & $.43^{* *}$ & $-.31^{* *}$ \\
\hline 6. Emotional suppression & $.25^{* *}$ & $-.20^{* *}$ & $.20^{* *}$ & $-.17^{* *}$ & $.17^{* *}$ & $.49^{* *}$ & -.01 & $.15^{* *}$ & $-.23^{* *}$ \\
\hline 7. Cognitive reappraisal & $-.17^{* *}$ & $.19^{* *}$ & $-.09^{* *}$ & $.16^{* *}$ & $-.15^{* *}$ & -.04 & $.41^{* *}$ & $-.18^{* *}$ & $.19^{* *}$ \\
\hline 8. Emotion control difficulties & $.49^{* *}$ & $-.30^{* *}$ & $.29^{* *}$ & $-.23^{* *}$ & $.41^{* *}$ & $.15^{* *}$ & $-.17^{* *}$ & $.51^{* *}$ & $-.27^{* *}$ \\
\hline 9. Perceived support & $-.36^{* *}$ & $.48^{* *}$ & $-.23^{* *}$ & $.27^{* *}$ & $-.29^{* *}$ & $-.20^{* *}$ & $.22^{* *}$ & $-.27^{* *}$ & $.65^{* *}$ \\
\hline
\end{tabular}

Note. Correlations for pre-lockdown measures are above the diagonal. Correlations across measures assessed during the lockdown are below the diagonal. Bold correlations on the diagonal represent the within-measure associations across pre-lockdown and lockdown assessments. ${ }^{*} p<.05 .{ }^{*} p<.01$. 


\section{Table 8.}

The Associations between Emotion Regulation Strategies and Psychological and Physical Health in Study 2

\begin{tabular}{|c|c|c|c|c|c|c|c|c|c|c|c|c|c|c|c|c|c|c|c|c|}
\hline \multirow[t]{2}{*}{ Predictors } & \multicolumn{5}{|c|}{ Psychological Distress } & \multicolumn{5}{|c|}{ Personal Well-being } & \multicolumn{5}{|c|}{ Fatigue (Low Energy) } & \multicolumn{5}{|c|}{ General Health } \\
\hline & $B$ & $95 \% C I$ & $t$ & $p$ & $\beta$ & $B$ & $95 \% C I$ & $t$ & $p$ & $\beta$ & $B$ & $95 \%$ CI & $t$ & $p$ & $\beta$ & $B$ & $95 \% C I$ & $t$ & $p$ & $\beta$ \\
\hline \multicolumn{21}{|c|}{ Emotion Regulation Strategies } \\
\hline Rumination & .29 & $.267, .315$ & 23.87 & $<.001$ & .43 & -.24 & $-.301,-.171$ & -7.12 & $<.001$ & -.13 & .24 & $.197, .287$ & 10.49 & $<.001$ & .22 & -.12 & $-.169,-.077$ & -5.24 & $<.001$ & -.10 \\
\hline $\begin{array}{l}\text { Emotional } \\
\text { suppression }\end{array}$ & .03 & $.020, .044$ & 5.04 & $<.001$ & .08 & -.04 & $-.078,-.006$ & -2.29 & .022 & -.04 & .06 & $.036, .086$ & 4.79 & $<.001$ & .10 & -.04 & $-.065,-.013$ & -2.93 & .003 & -.05 \\
\hline $\begin{array}{l}\text { Cognitive } \\
\text { reappraisal }\end{array}$ & -.02 & $-.036,-.008$ & -3.01 & $<.001$ & -.05 & .10 & $.053, .137$ & 4.46 & $<.001$ & .08 & -.01 & $-.043, .015$ & -0.94 & .347 & .02 & .05 & $.011, .081$ & 3.32 & .001 & .06 \\
\hline
\end{tabular}

Note. All analyses control for the pre-pandemic assessment of the health outcome assessed 1-year earlier along with the demographic covariates. See OSM for full details of the effect of demographic variables. Ns for these analyses varied from 1,595 to 1,607 due to small amounts of missing data in the health outcomes. 
Table 9.

Controlling for Alternative Explanations for the Links Between Emotion Regulation and Psychological and Physical Health in Study 2

\begin{tabular}{|c|c|c|c|c|c|c|c|c|c|c|c|c|c|c|c|c|c|c|c|c|}
\hline \multirow[t]{2}{*}{ Predictors } & \multicolumn{5}{|c|}{ Psychological Distress } & \multicolumn{5}{|c|}{ Personal Well-being } & \multicolumn{5}{|c|}{ Fatigue (Low Energy) } & \multicolumn{5}{|c|}{ General Health } \\
\hline & $B$ & $95 \% C I$ & $t$ & $p$ & $\beta$ & $B$ & $95 \% C I$ & $t$ & $p$ & $\beta$ & $B$ & $95 \% C I$ & $t$ & $p$ & $\beta$ & $B$ & $95 \% C I$ & $t$ & $p$ & $\beta$ \\
\hline \multicolumn{21}{|c|}{ Controlling for Emotion Control Difficulties } \\
\hline Rumination & .27 & $.242, .291$ & 21.55 & $<.001$ & .40 & -.20 & $-.266,-.129$ & -5.66 & $<.001$ & -.11 & .23 & $.177, .273$ & 9.22 & $<.001$ & .20 & -.10 & $-.150,-.052$ & -4.02 & $<.001$ & -.08 \\
\hline Emotional suppression & .03 & $.017, .042$ & 4.71 & $<.001$ & .08 & -.04 & $-.074,-.002$ & -2.08 & .038 & -.04 & .06 & $.033, .083$ & 4.52 & $<.001$ & .09 & -.04 & $-.062,-.010$ & -2.69 & .007 & -.05 \\
\hline Cognitive reappraisal & -.02 & $-.030,-.002$ & -2.17 & .030 & -.04 & .09 & $.044, .127$ & 4.01 & $<.001$ & .07 & -.01 & $-.039, .020$ & -0.64 & .523 & -.01 & .05 & $.016, .076$ & 2.99 & .003 & .06 \\
\hline $\begin{array}{l}\text { Emotion Control } \\
\text { Difficulties }\end{array}$ & .06 & $.041, .068$ & 7.94 & $<.001$ & .14 & -.07 & $-.111,-.032$ & -3.56 & $<.001$ & .07 & .03 & $.006, .060$ & 2.36 & .019 & .05 & .04 & $-.070,-.052$ & -2.85 & .004 & -.06 \\
\hline \multicolumn{21}{|c|}{ Controlling for Perceived Support } \\
\hline Rumination & .28 & $.254, .302$ & 22.81 & $<.001$ & .41 & -.17 & $-.231,-.103$ & -5.12 & $<.001$ & -.09 & .23 & $.180, .273$ & 9.58 & $<.001$ & .20 & -.10 & $-.150,-.055$ & -4.25 & $<.001$ & -.08 \\
\hline Emotional suppression & .03 & $.015, .040$ & 4.33 & $<.001$ & .07 & -.02 & $-.056, .014$ & -1.20 & .230 & -.02 & .06 & $.031, .081$ & 4.38 & $<.001$ & .09 & -.03 & $-.058,-.006$ & -2.45 & .014 & -.05 \\
\hline Cognitive reappraisal & -.01 & $-.028, .000$ & -1.93 & .054 & -.03 & .06 & $.021, .103$ & 2.98 & .003 & .05 & -.01 & $-.036, .023$ & -0.45 & .653 & -.01 & .04 & $.012, .073$ & 2.72 & .007 & .05 \\
\hline
\end{tabular}

Note. All analyses control for the pre-pandemic assessment of the health outcome assessed 1-year earlier along with the demographic covariates. 